\title{
Molecular composition and photochemical evolution of water-soluble organic carbon (WSOC) extracted from field biomass burning aerosols using high-resolution mass spectrometry
}

Jing Cai et al.

Correspondence to: Zhiqiang Yu (zhiqiang@gig.ac.cn)

The copyright of individual parts of the supplement might differ from the CC BY 4.0 License. 
Table S1: The general information for present biomass burning samples.

\begin{tabular}{|l|l|l|l|l|l|l|}
\hline $\begin{array}{l}\text { Sampling } \\
\text { site }\end{array}$ & Sample ID & $\begin{array}{l}\text { OC } \\
\left(\mathrm{mg} \mathrm{m}^{-3}\right)\end{array}$ & $\begin{array}{l}\text { EC } \\
\left(\mathrm{mg} \mathrm{m}^{-3}\right)\end{array}$ & $\begin{array}{l}\text { WSOC } \\
\left(\mathrm{mg} \mathrm{m}^{-3}\right)\end{array}$ & $\begin{array}{l}\mathrm{PM}_{2.5} \\
\left(\mathrm{mg} \mathrm{m}^{-3}\right)\end{array}$ & $\begin{array}{l}\text { Aqueous } \\
\text { Extract } \mathrm{pH}\end{array}$ \\
\hline \multirow{3}{*}{ HNWX } & HNWX-1 & 7.66 & 0.24 & 3.13 & 15.30 & 5.7 \\
\cline { 2 - 7 } & HNWX-2 & 7.38 & 0.40 & 2.58 & 20.24 & 6.2 \\
\cline { 2 - 7 } & HNWX-3 & 3.76 & 0.08 & 1.25 & 6.46 & 5.4 \\
\hline \multirow{3}{*}{ HBDM } & HBDM-1 & 15.24 & 0.19 & 5.39 & 28.03 & 5.0 \\
\cline { 2 - 7 } & HBDM-2 & 11.09 & 0.26 & 2.58 & 20.48 & 5.9 \\
\cline { 2 - 7 } & HBDM-3 & 11.26 & 0.25 & 5.13 & 21.47 & 4.5 \\
\hline
\end{tabular}

Table S2: The classification features of assigned compounds for analyzed extracts

\begin{tabular}{|l|l|l|l|l|l|l|}
\hline $\begin{array}{l}\text { Elemental } \\
\text { composition }\end{array}$ & Sample ID & $\begin{array}{l}\text { Number of } \\
\text { formulas }\end{array}$ & $\mathrm{OM} / \mathrm{OC}$ & $\mathrm{H} / \mathrm{C}$ & $\mathrm{O} / \mathrm{C}$ & $\mathrm{DBE}$ \\
\hline \multirow{4}{*}{ CHO } & HN-WX1 & 486 & $1.66 \pm 0.39$ & $1.26 \pm 0.38$ & $0.42 \pm 0.29$ & $5.4 \pm 2.8$ \\
\cline { 2 - 7 } & HN-WX2 & 453 & $1.59 \pm 0.34$ & $1.31 \pm 0.38$ & $0.36 \pm 0.25$ & $5.1 \pm 2.7$ \\
\cline { 2 - 7 } & HB-DM1 & 502 & $1.63 \pm 0.37$ & $1.26 \pm 0.37$ & $0.40 \pm 0.27$ & $5.5 \pm 2.3$ \\
\cline { 2 - 7 } & HB-DM2 & 499 & $1.57 \pm 0.32$ & $1.31 \pm 0.40$ & $0.34 \pm 0.24$ & $5.1 \pm 2.8$ \\
\hline \multirow{5}{*}{ CHOS } & HN-WX1 & 36 & $2.94 \pm 1.04$ & $1.79 \pm 0.58$ & $0.91 \pm 0.56$ & $2.0 \pm 2.1$ \\
\cline { 2 - 7 } & HN-WX2 & 23 & $3.29 \pm 1.26$ & $1.83 \pm 0.37$ & $1.16 \pm 0.70$ & $1.6 \pm 1.2$ \\
\cline { 2 - 7 } & HB-DM1 & 27 & $2.88 \pm 0.71$ & $1.65 \pm 0.65$ & $0.91 \pm 0.37$ & $2.5 \pm 1.4$ \\
\cline { 2 - 7 } & HB-DM2 & 11 & $2.93 \pm 1.20$ & $1.62 \pm 0.51$ & $0.98 \pm 0.64$ & $2.9 \pm 3.1$ \\
\hline \multirow{5}{*}{ CHON } & HN-WX1 & 262 & $1.65 \pm 0.26$ & $1.19 \pm 0.32$ & $0.29 \pm 0.15$ & $6.0 \pm 2.0$ \\
\cline { 2 - 7 } & HN-WX2 & 294 & $1.64 \pm 0.28$ & $1.23 \pm 0.35$ & $0.28 \pm 0.18$ & $5.7 \pm 2.2$ \\
\cline { 2 - 7 } & HB-DM1 & 326 & $1.62 \pm 0.26$ & $1.20 \pm 0.33$ & $0.28 \pm 0.17$ & $6.1 \pm 2.1$ \\
\cline { 2 - 7 } & HB-DM2 & 294 & $1.64 \pm 0.31$ & $1.21 \pm 0.34$ & $0.28 \pm 0.20$ & $5.8 \pm 2.2$ \\
\hline \multirow{5}{*}{ Other } & HN-WX1 & 19 & - & - & - & - \\
\cline { 2 - 7 } & HN-WX2 & 21 & - & - & - & - \\
\cline { 2 - 7 } & HB-DM1 & 32 & - & - & - & - \\
\cline { 2 - 7 } & HB-DM2 & 23 & - & - & - & - \\
\hline
\end{tabular}


Table S3: List of ion species with assigned formulas detected in present samples using HRMS.

\begin{tabular}{|c|c|c|c|c|c|}
\hline $\begin{array}{l}\text { Compound } \\
\text { class }\end{array}$ & $\begin{array}{l}\text { Measured } \\
\mathrm{m} / \mathrm{z}\end{array}$ & $\begin{array}{l}\text { Theoretical } \\
\mathrm{m} / \mathrm{z}\end{array}$ & $\begin{array}{l}\text { Relative Deviation } \\
\text { between } \\
\text { theoretical and } \\
\text { measured } \mathrm{m} / \mathrm{z} \\
(\mathrm{ppm})\end{array}$ & $\begin{array}{l}\text { DBE of } \\
{[\mathrm{M}-\mathrm{H}]^{-}}\end{array}$ & $\begin{array}{c}\text { Assigned } \\
\text { Formula [M- } \\
\mathrm{H}^{-}\end{array}$ \\
\hline \multirow{36}{*}{$\mathrm{CHO}$} & 55.0187 & 55.0189 & -4.76 & 2.5 & $\mathrm{C} 3 \mathrm{H} 3 \mathrm{O}$ \\
\hline & 57.0344 & 57.0346 & -3.96 & 1.5 & $\mathrm{C} 3 \mathrm{H} 5 \mathrm{O}$ \\
\hline & 59.0136 & 59.0139 & -3.55 & 1.5 & $\mathrm{C} 2 \mathrm{H} 3 \mathrm{O} 2$ \\
\hline & 67.0187 & 67.0189 & -2.97 & 3.5 & $\mathrm{C} 4 \mathrm{H} 3 \mathrm{O}$ \\
\hline & 69.0344 & 69.0346 & -2.96 & 2.5 & $\mathrm{C} 4 \mathrm{H} 5 \mathrm{O}$ \\
\hline & 71.0500 & 71.0502 & -2.76 & 1.5 & $\mathrm{C} 4 \mathrm{H} 7 \mathrm{O}$ \\
\hline & 72.9929 & 72.8831 & -2.55 & 2.5 & $\mathrm{C} 2 \mathrm{H} \mathrm{O} 3$ \\
\hline & 73.0293 & 73.0295 & -2.54 & 1.5 & $\mathrm{C} 3 \mathrm{H} 5 \mathrm{O} 2$ \\
\hline & 75.0086 & 75.0088 & -2.36 & 1.5 & $\mathrm{C} 2 \mathrm{H} 3 \mathrm{O} 3$ \\
\hline & 81.0344 & 81.0346 & -2.52 & 3.5 & $\mathrm{C} 5 \mathrm{H} 5 \mathrm{O}$ \\
\hline & 83.0137 & 83.0139 & -2.17 & 3.5 & $\mathrm{C} 4 \mathrm{H} 3 \mathrm{O} 2$ \\
\hline & 85.0293 & 85.0295 & -1.86 & 2.5 & $\mathrm{C} 4 \mathrm{H} 5 \mathrm{O} 2$ \\
\hline & 85.0657 & 85.0659 & -2.36 & 1.5 & $\mathrm{C} 5 \mathrm{H} 9 \mathrm{O}$ \\
\hline & 87.0450 & 87.0452 & -1.84 & 1.5 & $\mathrm{C} 4 \mathrm{H} 7 \mathrm{O} 2$ \\
\hline & 88.9879 & 88.9880 & -1.70 & 1.5 & $\mathrm{C} 2 \mathrm{H} \mathrm{O} 4$ \\
\hline & 91.0399 & 91.0401 & -1.80 & 0.5 & $\mathrm{C} 3 \mathrm{H} 7 \mathrm{O} 3$ \\
\hline & 93.0345 & 93.0346 & -1.36 & 4.5 & C6 H5 O \\
\hline & 95.0137 & 95.0139 & -1.54 & 4.5 & $\mathrm{C} 5 \mathrm{H} 3 \mathrm{O} 2$ \\
\hline & 95.0501 & 95.0502 & -1.77 & 3.5 & C6 H7 O \\
\hline & 97.0294 & 97.0295 & -1.54 & 3.5 & $\mathrm{C} 5 \mathrm{H} 5 \mathrm{O} 2$ \\
\hline & 97.0657 & 97.0659 & -1.76 & 2.5 & C6 H9 O \\
\hline & 99.0086 & 99.0088 & -1.97 & 3.5 & $\mathrm{C} 4 \mathrm{H} 3 \mathrm{O} 3$ \\
\hline & 99.0450 & 99.0452 & -1.51 & 2.5 & $\mathrm{C} 5 \mathrm{H} 7 \mathrm{O} 2$ \\
\hline & 99.0814 & 99.0815 & -1.93 & 1.5 & C6 H11 O \\
\hline & 101.0243 & 101.0244 & -1.29 & 2.5 & $\mathrm{C} 4 \mathrm{H} 5 \mathrm{O} 3$ \\
\hline & 103.0399 & 103.0401 & -1.24 & 1.5 & $\mathrm{C} 4 \mathrm{H} 7 \mathrm{O} 3$ \\
\hline & 107.0501 & 107.0502 & -1.20 & 4.5 & $\mathrm{C} 7 \mathrm{H} 7 \mathrm{O}$ \\
\hline & 109.0293 & 109.0295 & -1.51 & 4.5 & C6 H5 O2 \\
\hline & 109.0658 & 109.0659 & -1.14 & 3.5 & C7 H9 O \\
\hline & 111.0087 & 111.0088 & -1.06 & 4.5 & $\mathrm{C} 5 \mathrm{H} 3 \mathrm{O} 3$ \\
\hline & 111.0450 & 111.0452 & -1.21 & 3.5 & $\mathrm{C} 6 \mathrm{H} 7 \mathrm{O} 2$ \\
\hline & 113.0243 & 113.0244 & -1.07 & 3.5 & $\mathrm{C} 5 \mathrm{H} 5 \mathrm{O} 3$ \\
\hline & 113.0607 & 113.0608 & -0.96 & 2.5 & $\mathrm{C} 6 \mathrm{H} 9 \mathrm{O} 2$ \\
\hline & 115.0399 & 115.0401 & -1.31 & 2.5 & $\mathrm{C} 5 \mathrm{H} 7 \mathrm{O} 3$ \\
\hline & 115.0763 & 115.0765 & -1.15 & 1.5 & C6 H11 O2 \\
\hline & 117.0191 & 117.0193 & -1.63 & 2.5 & C4 H5 O4 \\
\hline
\end{tabular}




\begin{tabular}{|c|c|c|c|c|}
\hline 117.0344 & 117.0346 & -1.40 & 6.5 & $\mathrm{C} 8 \mathrm{H} 5 \mathrm{O}$ \\
\hline 117.0556 & 117.0557 & -0.99 & 1.5 & C5 H9 O3 \\
\hline 119.0349 & 119.0350 & -0.95 & 1.5 & $\mathrm{C} 4 \mathrm{H} 7 \mathrm{O} 4$ \\
\hline 119.0501 & 119.0502 & -0.99 & 5.5 & $\mathrm{C} 8 \mathrm{H} 7 \mathrm{O}$ \\
\hline 121.0294 & 121.0295 & -1.08 & 5.5 & $\mathrm{C} 7 \mathrm{H} 5 \mathrm{O} 2$ \\
\hline 121.0658 & 121.0659 & -0.84 & 4.5 & $\mathrm{C} 8 \mathrm{H} 9 \mathrm{O}$ \\
\hline 123.0087 & 123.0088 & -0.74 & 5.5 & $\mathrm{C} 6 \mathrm{H} 3 \mathrm{O} 3$ \\
\hline 123.0450 & 123.0452 & -1.01 & 4.5 & $\mathrm{C} 7 \mathrm{H} 7 \mathrm{O} 2$ \\
\hline 123.0814 & 123.0815 & -0.94 & 3.5 & C8 H11 O \\
\hline 125.0243 & 125.0244 & -0.77 & 4.5 & C6 H5 O3 \\
\hline 125.0607 & 125.0608 & -0.89 & 3.5 & $\mathrm{C} 7 \mathrm{H} 9 \mathrm{O} 2$ \\
\hline 125.0970 & 125.0972 & -1.83 & 2.5 & $\mathrm{C} 8 \mathrm{H} 13 \mathrm{O}$ \\
\hline 127.0036 & 127.0037 & -0.89 & 4.5 & $\mathrm{C} 5 \mathrm{H} 3 \mathrm{O} 4$ \\
\hline 127.0400 & 127.0401 & -0.66 & 3.5 & C6 H7 O3 \\
\hline 127.0764 & 127.0765 & -0.74 & 2.5 & $\mathrm{C} 7 \mathrm{H} 11 \mathrm{O} 2$ \\
\hline 129.0192 & 129.0193 & -1.27 & 3.5 & $\mathrm{C} 5 \mathrm{H} 5 \mathrm{O} 4$ \\
\hline 129.0556 & 129.0557 & -0.74 & 2.5 & C6 H9 O3 \\
\hline 129.0920 & 129.0921 & -0.65 & 1.5 & $\mathrm{C} 7 \mathrm{H} 13 \mathrm{O} 2$ \\
\hline 131.0349 & 131.0350 & -0.68 & 2.5 & $\mathrm{C} 5 \mathrm{H} 7 \mathrm{O} 4$ \\
\hline 131.0501 & 131.0502 & -0.97 & 6.5 & C9 H7 O \\
\hline 131.0713 & 131.0714 & -0.67 & 1.5 & C6 H11 O3 \\
\hline 133.0141 & 133.0143 & -0.95 & 2.5 & $\mathrm{C} 4 \mathrm{H} 5 \mathrm{O} 5$ \\
\hline 133.0294 & 133.0295 & -0.54 & 6.5 & $\mathrm{C} 8 \mathrm{H} 5 \mathrm{O} 2$ \\
\hline 133.0505 & 133.0506 & -0.86 & 1.5 & $\mathrm{C} 5 \mathrm{H} 9 \mathrm{O} 4$ \\
\hline 133.0658 & 133.0659 & -0.78 & 5.5 & C9 H9 O \\
\hline 135.0451 & 135.0452 & -0.69 & 5.5 & $\mathrm{C} 8 \mathrm{H} 7 \mathrm{O} 2$ \\
\hline 135.0815 & 135.0815 & -0.48 & 4.5 & C9 H11 O \\
\hline 137.0243 & 137.0244 & -0.58 & 5.5 & $\mathrm{C} 7 \mathrm{H} 5 \mathrm{O} 3$ \\
\hline 137.0607 & 137.0608 & -0.74 & 4.5 & $\mathrm{C} 8 \mathrm{H} 9 \mathrm{O} 2$ \\
\hline 137.0971 & 137.0972 & -1.00 & 3.5 & C9 H13 O \\
\hline 139.0036 & 139.0037 & -0.79 & 5.5 & C6 H3 O4 \\
\hline 139.0400 & 139.0401 & -0.50 & 4.5 & $\mathrm{C} 7 \mathrm{H} 7 \mathrm{O} 3$ \\
\hline 139.0764 & 139.0765 & -0.62 & 3.5 & $\mathrm{C} 8 \mathrm{H} 11 \mathrm{O} 2$ \\
\hline 141.0193 & 141.0193 & -0.36 & 4.5 & $\mathrm{C} 6 \mathrm{H} 5 \mathrm{O} 4$ \\
\hline 141.0556 & 141.0557 & -0.52 & 3.5 & $\mathrm{C} 7 \mathrm{H} 9 \mathrm{O} 3$ \\
\hline 141.0921 & 141.0921 & -0.30 & 2.5 & $\mathrm{C} 8 \mathrm{H} 13 \mathrm{O} 2$ \\
\hline 143.0349 & 143.0350 & -0.46 & 3.5 & $\mathrm{C} 6 \mathrm{H} 7 \mathrm{O} 4$ \\
\hline 143.0502 & 143.0502 & -0.44 & 7.5 & $\mathrm{C} 10 \mathrm{H} 7 \mathrm{O}$ \\
\hline 143.0713 & 143.0714 & -0.46 & 2.5 & C7 H11 O3 \\
\hline 143.1077 & 143.1078 & -0.56 & 1.5 & $\mathrm{C} 8 \mathrm{H} 15 \mathrm{O} 2$ \\
\hline 145.0142 & 145.0143 & -0.44 & 3.5 & C5 H5 O5 \\
\hline 145.0294 & 145.0295 & -0.73 & 7.5 & $\mathrm{C} 9 \mathrm{H} 5 \mathrm{O} 2$ \\
\hline 145.0658 & 145.0659 & -0.45 & 6.5 & $\mathrm{C} 10 \mathrm{H} 9 \mathrm{O}$ \\
\hline
\end{tabular}




\begin{tabular}{|c|c|c|c|c|}
\hline 147.0298 & 147.0299 & -0.50 & 2.5 & $\mathrm{C} 5 \mathrm{H} 7 \mathrm{O} 5$ \\
\hline 147.0451 & 147.0452 & -0.43 & 6.5 & $\mathrm{C} 9 \mathrm{H} 7 \mathrm{O} 2$ \\
\hline 147.0662 & 147.0663 & -0.74 & 1.5 & C6 H11 O4 \\
\hline 147.0815 & 147.0815 & -0.53 & 5.5 & C10 H11 O \\
\hline 149.0094 & 149.0095 & 1.27 & 2.5 & C4 H5 O6 \\
\hline 149.0454 & 149.0456 & -0.70 & 1.5 & C5 H9 O5 \\
\hline 149.0607 & 149.0608 & -0.79 & 5.5 & $\mathrm{C} 9 \mathrm{H} 9 \mathrm{O} 2$ \\
\hline 149.0971 & 149.0972 & -0.61 & 4.5 & $\mathrm{C} 10 \mathrm{H} 13 \mathrm{O}$ \\
\hline 150.9887 & 150.9888 & 2.08 & 2.5 & $\mathrm{C} 3 \mathrm{H} 3 \mathrm{O} 7$ \\
\hline 151.0036 & 151.0037 & -0.56 & 6.5 & $\mathrm{C} 7 \mathrm{H} 3 \mathrm{O} 4$ \\
\hline 151.0400 & 151.0401 & -0.44 & 5.5 & $\mathrm{C} 8 \mathrm{H} 7 \mathrm{O} 3$ \\
\hline 151.0611 & 151.0612 & -0.56 & 0.5 & $\mathrm{C} 5 \mathrm{H} 11 \mathrm{O} 5$ \\
\hline 151.0764 & 151.0765 & -0.45 & 4.5 & $\mathrm{C} 9 \mathrm{H} 11 \mathrm{O} 2$ \\
\hline 151.1128 & 151.1128 & -0.23 & 3.5 & $\mathrm{C} 10 \mathrm{H} 15 \mathrm{O}$ \\
\hline 153.0193 & 153.0193 & -0.28 & 5.5 & C7 H5 O4 \\
\hline 153.0557 & 153.0557 & -0.32 & 4.5 & $\mathrm{C} 8 \mathrm{H} 9 \mathrm{O} 3$ \\
\hline 153.0921 & 153.0921 & -0.18 & 3.5 & $\mathrm{C} 9 \mathrm{H} 13 \mathrm{O} 2$ \\
\hline 154.9985 & 154.9986 & -0.89 & 5.5 & C6 H3 O5 \\
\hline 155.0349 & 155.0350 & -0.40 & 4.5 & $\mathrm{C} 7 \mathrm{H} 7 \mathrm{O} 4$ \\
\hline 155.0714 & 155.0714 & -0.12 & 3.5 & $\mathrm{C} 8 \mathrm{H} 11 \mathrm{O} 3$ \\
\hline 155.1077 & 155.1078 & -0.18 & 2.5 & $\mathrm{C} 9 \mathrm{H} 15 \mathrm{O} 2$ \\
\hline 157.0142 & 157.0143 & -0.13 & 4.5 & C6 H5 O5 \\
\hline 157.0505 & 157.0506 & -0.87 & 3.5 & $\mathrm{C} 7 \mathrm{H} 9 \mathrm{O} 4$ \\
\hline 157.0659 & 157.0659 & -0.01 & 7.5 & C11 H9 O \\
\hline 157.1234 & 157.1234 & -0.36 & 1.5 & $\mathrm{C} 9 \mathrm{H} 17 \mathrm{O} 2$ \\
\hline 159.0298 & 159.0299 & -0.34 & 3.5 & C6 H7 O5 \\
\hline 159.0451 & 159.0452 & -0.48 & 7.5 & $\mathrm{C} 10 \mathrm{H} 7 \mathrm{O} 2$ \\
\hline 159.0662 & 159.0663 & -0.65 & 2.5 & C7 H11 O4 \\
\hline 159.0815 & 159.0815 & -0.30 & 6.5 & C11 H11 O \\
\hline 161.0093 & 161.0092 & 0.54 & 3.5 & C5 H5 O6 \\
\hline 161.0244 & 161.0244 & -0.12 & 7.5 & C9 H5 O3 \\
\hline 161.0455 & 161.0456 & -0.36 & 2.5 & C6 H9 O5 \\
\hline 161.0608 & 161.0608 & -0.30 & 6.5 & $\mathrm{C} 10 \mathrm{H} 9 \mathrm{O} 2$ \\
\hline 161.0819 & 161.0819 & -0.35 & 1.5 & C7 H13 O4 \\
\hline 161.0971 & 161.0972 & -0.39 & 5.5 & C11 H13 O \\
\hline 163.0035 & 163.0037 & -1.30 & 7.5 & $\mathrm{C} 8 \mathrm{H} 3 \mathrm{O} 4$ \\
\hline 163.0248 & 163.0248 & -0.19 & 2.5 & $\mathrm{C} 5 \mathrm{H} 7 \mathrm{O} 6$ \\
\hline 163.0400 & 163.0401 & -0.27 & 6.5 & $\mathrm{C} 9 \mathrm{H} 7 \mathrm{O} 3$ \\
\hline 163.0611 & 163.0612 & -0.34 & 1.5 & C6 H11 O5 \\
\hline 163.0764 & 163.0765 & -0.28 & 5.5 & $\mathrm{C} 10 \mathrm{H} 11 \mathrm{O} 2$ \\
\hline 163.1129 & 163.1128 & 0.15 & 4.5 & C11 H15 O \\
\hline 165.0193 & 165.0193 & -0.49 & 6.5 & $\mathrm{C} 8 \mathrm{H} 5 \mathrm{O} 4$ \\
\hline 165.0407 & 165.0408 & 1.39 & 1.5 & C5 H9 O6 \\
\hline
\end{tabular}




\begin{tabular}{|c|c|c|c|c|}
\hline 165.0556 & 165.0557 & -0.46 & 5.5 & C9 H9 O3 \\
\hline 165.0921 & 165.0921 & -0.15 & 4.5 & $\mathrm{C} 10 \mathrm{H} 13 \mathrm{O} 2$ \\
\hline 166.9986 & 166.9986 & 0.06 & 6.5 & C7 H3 O5 \\
\hline 167.0350 & 167.0350 & -0.20 & 5.5 & $\mathrm{C} 8 \mathrm{H} 7 \mathrm{O} 4$ \\
\hline 167.0714 & 167.0714 & -0.09 & 4.5 & C9 H11 O3 \\
\hline 167.1077 & 167.1078 & -0.07 & 3.5 & $\mathrm{C} 10 \mathrm{H} 15 \mathrm{O} 2$ \\
\hline 168.9994 & 168.9993 & 2.42 & 1.5 & C3 H5 O8 \\
\hline 169.0142 & 169.0143 & -0.32 & 5.5 & $\mathrm{C} 7 \mathrm{H} 5 \mathrm{O} 5$ \\
\hline 169.0506 & 169.0506 & -0.31 & 4.5 & $\mathrm{C} 8 \mathrm{H} 9 \mathrm{O} 4$ \\
\hline 169.0658 & 169.0659 & -0.29 & 8.5 & C12 H9 O \\
\hline 169.0870 & 169.0870 & -0.04 & 3.5 & C9 H13 O3 \\
\hline 169.1234 & 169.1234 & -0.07 & 2.5 & $\mathrm{C} 10 \mathrm{H} 17 \mathrm{O} 2$ \\
\hline 171.0299 & 171.0299 & -0.12 & 4.5 & $\mathrm{C} 7 \mathrm{H} 7 \mathrm{O} 5$ \\
\hline 171.0452 & 171.0452 & 0.09 & 8.5 & $\mathrm{C} 11 \mathrm{H} 7 \mathrm{O} 2$ \\
\hline 171.0662 & 171.0663 & -0.51 & 3.5 & $\mathrm{C} 8 \mathrm{H} 11 \mathrm{O} 4$ \\
\hline 171.0816 & 171.0815 & 0.07 & 7.5 & $\mathrm{C} 12 \mathrm{H} 11 \mathrm{O}$ \\
\hline 173.0455 & 173.0456 & -0.25 & 3.5 & C7 H9 O5 \\
\hline 173.0608 & 173.0608 & 0.09 & 7.5 & $\mathrm{C} 11 \mathrm{H} 9 \mathrm{O} 2$ \\
\hline 173.0972 & 173.0972 & -0.05 & 6.5 & $\mathrm{C} 12 \mathrm{H} 13 \mathrm{O}$ \\
\hline 175.0249 & 175.0248 & 0.23 & 3.5 & C6 H7 O6 \\
\hline 175.0401 & 175.0401 & -0.03 & 7.5 & $\mathrm{C} 10 \mathrm{H} 7 \mathrm{O} 3$ \\
\hline 175.0612 & 175.0612 & -0.12 & 2.5 & C7 H11 O5 \\
\hline 175.0765 & 175.0765 & -0.02 & 6.5 & C11 H11 O2 \\
\hline 175.0975 & 175.0976 & -0.29 & 1.5 & $\mathrm{C} 8 \mathrm{H} 15 \mathrm{O} 4$ \\
\hline 175.1128 & 175.1128 & -0.31 & 5.5 & $\mathrm{C} 12 \mathrm{H} 15 \mathrm{O}$ \\
\hline 177.0194 & 177.0193 & 0.11 & 7.5 & C9 H5 O4 \\
\hline 177.0405 & 177.0405 & 0.05 & 2.5 & C6 H9 O6 \\
\hline 177.0557 & 177.0557 & -0.05 & 6.5 & $\mathrm{C} 10 \mathrm{H} 9 \mathrm{O} 3$ \\
\hline 177.0921 & 177.0921 & 0.13 & 5.5 & $\mathrm{C} 11 \mathrm{H} 13 \mathrm{O} 2$ \\
\hline 179.0350 & 179.0350 & 0.14 & 6.5 & C9 H7 O4 \\
\hline 179.0561 & 179.0561 & -0.22 & 1.5 & C6 H11 O6 \\
\hline 179.0714 & 179.0714 & -0.04 & 5.5 & $\mathrm{C} 10 \mathrm{H} 11 \mathrm{O} 3$ \\
\hline 179.1078 & 179.1078 & 0.12 & 4.5 & C11 H15 O2 \\
\hline 181.0143 & 181.0143 & 0.42 & 6.5 & $\mathrm{C} 8 \mathrm{H} 5 \mathrm{O} 5$ \\
\hline 181.0506 & 181.0506 & -0.33 & 5.5 & C9 H9 O4 \\
\hline 181.0658 & 181.0659 & -0.65 & 9.5 & $\mathrm{C} 13 \mathrm{H} 9 \mathrm{O}$ \\
\hline 181.0718 & 181.0718 & 0.20 & 0.5 & C6 H13 O6 \\
\hline 181.0870 & 181.0870 & 0.06 & 4.5 & $\mathrm{C} 10 \mathrm{H} 13 \mathrm{O} 3$ \\
\hline 181.1234 & 181.1234 & 0.22 & 3.5 & $\mathrm{C} 11 \mathrm{H} 17 \mathrm{O} 2$ \\
\hline 183.0299 & 183.0299 & 0.21 & 5.5 & $\mathrm{C} 8 \mathrm{H} 7 \mathrm{O} 5$ \\
\hline 183.0451 & 183.0452 & -0.26 & 9.5 & $\mathrm{C} 12 \mathrm{H} 7 \mathrm{O} 2$ \\
\hline 183.0663 & 183.0663 & 0.21 & 4.5 & $\mathrm{C} 9 \mathrm{H} 11 \mathrm{O} 4$ \\
\hline 183.0815 & 183.0815 & 0.01 & 8.5 & $\mathrm{C} 13 \mathrm{H} 11 \mathrm{O}$ \\
\hline
\end{tabular}




\begin{tabular}{|c|c|c|c|c|}
\hline 183.1028 & 183.1027 & 0.45 & 3.5 & $\mathrm{C} 10 \mathrm{H} 15 \mathrm{O} 3$ \\
\hline 183.1391 & 183.1391 & 0.38 & 2.5 & C11 H19 O2 \\
\hline 185.0456 & 185.0456 & 0.13 & 4.5 & C8 H9 O5 \\
\hline 185.0609 & 185.0609 & 0.53 & 8.5 & $\mathrm{C} 12 \mathrm{H} 9 \mathrm{O} 2$ \\
\hline 185.0820 & 185.0819 & 0.39 & 3.5 & C9 H13 O4 \\
\hline 185.0972 & 185.0972 & 0.09 & 7.5 & $\mathrm{C} 13 \mathrm{H} 13 \mathrm{O}$ \\
\hline 185.1184 & 185.1183 & 0.44 & 2.5 & $\mathrm{C} 10 \mathrm{H} 17 \mathrm{O} 3$ \\
\hline 187.0248 & 187.0248 & 0.06 & 4.5 & $\mathrm{C} 7 \mathrm{H} 7 \mathrm{O} 6$ \\
\hline 187.0401 & 187.0401 & 0.13 & 8.5 & $\mathrm{C} 11 \mathrm{H} 7 \mathrm{O} 3$ \\
\hline 187.0612 & 187.0612 & 0.06 & 3.5 & C8 H11 O5 \\
\hline 187.0765 & 187.0765 & 0.36 & 7.5 & $\mathrm{C} 12 \mathrm{H} 11 \mathrm{O} 2$ \\
\hline 187.1128 & 187.1128 & -0.08 & 6.5 & $\mathrm{C} 13 \mathrm{H} 15 \mathrm{O}$ \\
\hline 189.0194 & 189.0193 & 0.15 & 8.5 & $\mathrm{C} 10 \mathrm{H} 5 \mathrm{O} 4$ \\
\hline 189.0405 & 189.0405 & 0.36 & 3.5 & C7 H9 O6 \\
\hline 189.0558 & 189.0557 & 0.25 & 7.5 & $\mathrm{C} 11 \mathrm{H} 9 \mathrm{O} 3$ \\
\hline 189.0769 & 189.0769 & 0.28 & 2.5 & $\mathrm{C} 8 \mathrm{H} 13 \mathrm{O} 5$ \\
\hline 189.0922 & 189.0921 & 0.49 & 6.5 & $\mathrm{C} 12 \mathrm{H} 13 \mathrm{O} 2$ \\
\hline 189.1133 & 189.1132 & 0.32 & 1.5 & C9 H17 O4 \\
\hline 191.0198 & 191.0198 & 0.39 & 3.5 & $\mathrm{C} 6 \mathrm{H} 7 \mathrm{O} 7$ \\
\hline 191.0349 & 191.0350 & -0.24 & 7.5 & $\mathrm{C} 10 \mathrm{H} 7 \mathrm{O} 4$ \\
\hline 191.0561 & 191.0561 & -0.03 & 2.5 & C7 H11 O6 \\
\hline 191.0714 & 191.0714 & 0.24 & 6.5 & $\mathrm{C} 11 \mathrm{H} 11 \mathrm{O} 3$ \\
\hline 191.1078 & 191.1078 & 0.34 & 5.5 & $\mathrm{C} 12 \mathrm{H} 15 \mathrm{O} 2$ \\
\hline 193.0144 & 193.0143 & 0.91 & 7.5 & C9 H5 O5 \\
\hline 193.0506 & 193.0506 & 0.00 & 6.5 & $\mathrm{C} 10 \mathrm{H} 9 \mathrm{O} 4$ \\
\hline 193.0659 & 193.0659 & -0.19 & 10.5 & $\mathrm{C} 14 \mathrm{H} 9 \mathrm{O}$ \\
\hline 193.0720 & 193.0721 & 0.98 & 1.5 & C7 H13 O6 \\
\hline 193.0871 & 193.0870 & 0.32 & 5.5 & $\mathrm{C} 11 \mathrm{H} 13 \mathrm{O} 3$ \\
\hline 193.1235 & 193.1234 & 0.24 & 4.5 & $\mathrm{C} 12 \mathrm{H} 17 \mathrm{O} 2$ \\
\hline 195.0300 & 195.0299 & 0.61 & 6.5 & C9 H7 O5 \\
\hline 195.0452 & 195.0452 & 0.15 & 10.5 & $\mathrm{C} 13 \mathrm{H} 7 \mathrm{O} 2$ \\
\hline 195.0512 & 195.0511 & 0.94 & 1.5 & C6 H11 O7 \\
\hline 195.0663 & 195.0663 & 0.12 & 5.5 & C10 H11 O4 \\
\hline 195.0815 & 195.0815 & -0.08 & 9.5 & C14 H11 O \\
\hline 195.1027 & 195.1027 & 0.35 & 4.5 & $\mathrm{C} 11 \mathrm{H} 15 \mathrm{O} 3$ \\
\hline 195.1391 & 195.1391 & 0.40 & 3.5 & $\mathrm{C} 12 \mathrm{H} 19 \mathrm{O} 2$ \\
\hline 197.0457 & 197.0456 & 0.60 & 5.5 & C9 H9 O5 \\
\hline 197.0609 & 197.0609 & 0.44 & 9.5 & $\mathrm{C} 13 \mathrm{H} 9 \mathrm{O} 2$ \\
\hline 197.0820 & 197.0819 & 0.54 & 4.5 & $\mathrm{C} 10 \mathrm{H} 13 \mathrm{O} 4$ \\
\hline 197.0973 & 197.0973 & 0.62 & 5.5 & $\mathrm{C} 14 \mathrm{H} 13 \mathrm{O}$ \\
\hline 197.1184 & 197.1183 & 0.30 & 3.5 & C11 H17 O3 \\
\hline 197.1548 & 197.1547 & 0.52 & 2.5 & $\mathrm{C} 12 \mathrm{H} 21 \mathrm{O} 2$ \\
\hline 199.0403 & 199.0401 & 1.07 & 9.5 & $\mathrm{C} 12 \mathrm{H} 7 \mathrm{O} 3$ \\
\hline
\end{tabular}




\begin{tabular}{|c|c|c|c|c|}
\hline 199.0614 & 199.0612 & 0.78 & 4.5 & C9 H11 O5 \\
\hline 199.0765 & 199.0765 & 0.39 & 8.5 & $\mathrm{C} 13 \mathrm{H} 11 \mathrm{O} 2$ \\
\hline 199.0977 & 199.0976 & 0.63 & 3.5 & $\mathrm{C} 10 \mathrm{H} 15 \mathrm{O} 4$ \\
\hline 199.1341 & 199.1340 & 0.52 & 2.5 & $\mathrm{C} 11 \mathrm{H} 19 \mathrm{O} 3$ \\
\hline 199.1703 & 199.1704 & -0.38 & 1.5 & $\mathrm{C} 12 \mathrm{H} 23 \mathrm{O} 2$ \\
\hline 201.0407 & 201.0408 & 1.04 & 4.5 & C8 H9 O6 \\
\hline 201.0559 & 201.0558 & 0.66 & 8.5 & C12 H9 O3 \\
\hline 201.0770 & 201.0769 & 0.95 & 3.5 & C9 H13 O5 \\
\hline 201.0922 & 201.0921 & 0.70 & 7.5 & $\mathrm{C} 13 \mathrm{H} 13 \mathrm{O} 2$ \\
\hline 203.0351 & 203.0350 & 0.38 & 8.5 & $\mathrm{C} 11 \mathrm{H} 7 \mathrm{O} 4$ \\
\hline 203.0561 & 203.0561 & 0.08 & 3.5 & C8 H11 O6 \\
\hline 203.0714 & 203.0714 & 0.26 & 7.5 & $\mathrm{C} 12 \mathrm{H} 11 \mathrm{O} 3$ \\
\hline 203.0927 & 203.0925 & 0.78 & 2.5 & C9 H15 O5 \\
\hline 203.1078 & 203.1078 & 0.44 & 6.5 & $\mathrm{C} 13 \mathrm{H} 15 \mathrm{O} 2$ \\
\hline 205.0143 & 205.0143 & 0.41 & 8.5 & $\mathrm{C} 10 \mathrm{H} 5 \mathrm{O} 5$ \\
\hline 205.0354 & 205.0354 & 0.00 & 3.5 & C7 H9 O7 \\
\hline 205.0505 & 205.0506 & -0.75 & 7.5 & C11 H9 O4 \\
\hline 205.0718 & 205.0718 & 0.13 & 2.5 & C8 H13 O6 \\
\hline 205.0871 & 205.0870 & 0.40 & 6.5 & $\mathrm{C} 12 \mathrm{H} 13 \mathrm{O} 3$ \\
\hline 205.1235 & 205.1234 & 0.53 & 5.5 & $\mathrm{C} 13 \mathrm{H} 17 \mathrm{O} 2$ \\
\hline 207.0300 & 207.0299 & 0.68 & 7.5 & $\mathrm{C} 10 \mathrm{H} 7 \mathrm{O} 5$ \\
\hline 207.0510 & 207.0510 & 0.01 & 2.5 & C7 H11 O7 \\
\hline 207.0664 & 207.0663 & 0.33 & 6.5 & $\mathrm{C} 11 \mathrm{H} 11 \mathrm{O} 4$ \\
\hline 207.1027 & 207.1027 & 0.24 & 5.5 & $\mathrm{C} 12 \mathrm{H} 15 \mathrm{O} 3$ \\
\hline 207.1391 & 207.1391 & 0.25 & 4.5 & $\mathrm{C} 13 \mathrm{H} 19 \mathrm{O} 2$ \\
\hline 209.0457 & 209.0456 & 0.52 & 6.5 & C10 H9 O5 \\
\hline 209.0609 & 209.0609 & 0.32 & 10.5 & $\mathrm{C} 14 \mathrm{H} 9 \mathrm{O} 2$ \\
\hline 209.0820 & 209.0819 & 0.35 & 5.5 & $\mathrm{C} 11 \mathrm{H} 13 \mathrm{O} 4$ \\
\hline 209.0973 & 209.0973 & 0.49 & 9.5 & C15 H13 O \\
\hline 209.1183 & 209.1183 & -0.11 & 4.5 & $\mathrm{C} 12 \mathrm{H} 17 \mathrm{O} 3$ \\
\hline 209.1548 & 209.1547 & 0.52 & 3.5 & $\mathrm{C} 13 \mathrm{H} 21 \mathrm{O} 2$ \\
\hline 211.0248 & 211.0248 & 0.08 & 6.5 & C9 H7 O6 \\
\hline 211.0401 & 211.0401 & 0.25 & 10.5 & $\mathrm{C} 13 \mathrm{H} 7 \mathrm{O} 3$ \\
\hline 211.0613 & 211.0612 & 0.45 & 5.5 & C10 H11 O5 \\
\hline 211.0765 & 211.0765 & 0.22 & 9.5 & $\mathrm{C} 14 \mathrm{H} 11 \mathrm{O} 2$ \\
\hline 211.0978 & 211.0976 & 0.87 & 4.5 & $\mathrm{C} 11 \mathrm{H} 15 \mathrm{O} 4$ \\
\hline 211.1340 & 211.1340 & -0.04 & 3.5 & $\mathrm{C} 12 \mathrm{H} 19 \mathrm{O} 3$ \\
\hline 211.1705 & 211.1704 & 0.48 & 2.5 & $\mathrm{C} 13 \mathrm{H} 23 \mathrm{O} 2$ \\
\hline 213.0406 & 213.0405 & 0.57 & 5.5 & C9 H9 O6 \\
\hline 213.0557 & 213.0557 & 0.00 & 9.5 & $\mathrm{C} 13 \mathrm{H} 9 \mathrm{O} 3$ \\
\hline 213.0769 & 213.0769 & 0.21 & 4.5 & $\mathrm{C} 10 \mathrm{H} 13 \mathrm{O}$ \\
\hline 213.0921 & 213.0921 & -0.18 & 8.5 & $\mathrm{C} 14 \mathrm{H} 13 \mathrm{O} 2$ \\
\hline 213.1132 & 213.1132 & 0.04 & 3.5 & $\mathrm{C} 11 \mathrm{H} 17 \mathrm{O} 4$ \\
\hline
\end{tabular}




\begin{tabular}{|c|c|c|c|c|}
\hline 213.1497 & 213.1496 & 0.14 & 2.5 & $\mathrm{C} 12 \mathrm{H} 21 \mathrm{O} 3$ \\
\hline 215.0352 & 215.0350 & 0.97 & 9.5 & $\mathrm{C} 12 \mathrm{H} 7 \mathrm{O} 4$ \\
\hline 215.0562 & 215.0561 & 0.17 & 4.5 & C9 H11 O6 \\
\hline 215.0715 & 215.0714 & 0.52 & 8.5 & $\mathrm{C} 13 \mathrm{H} 11 \mathrm{O} 3$ \\
\hline 215.0926 & 215.0925 & 0.40 & 3.5 & $\mathrm{C} 10 \mathrm{H} 15 \mathrm{O}$ \\
\hline 215.1077 & 215.1078 & -0.20 & 7.5 & $\mathrm{C} 14 \mathrm{H} 15 \mathrm{O} 2$ \\
\hline 215.1655 & 215.1653 & 0.93 & 1.5 & $\mathrm{C} 12 \mathrm{H} 23 \mathrm{O} 3$ \\
\hline 217.0508 & 217.0507 & 0.68 & 8.5 & $\mathrm{C} 12 \mathrm{H} 9 \mathrm{O} 4$ \\
\hline 217.0719 & 217.0718 & 0.52 & 3.5 & C9 H13 O6 \\
\hline 217.0872 & 217.0870 & 0.60 & 7.5 & $\mathrm{C} 13 \mathrm{H} 13 \mathrm{O} 3$ \\
\hline 217.1082 & 217.1082 & 0.36 & 2.5 & $\mathrm{C} 10 \mathrm{H} 17 \mathrm{O5}$ \\
\hline 217.1234 & 217.1234 & 0.06 & 6.5 & $\mathrm{C} 14 \mathrm{H} 17 \mathrm{O} 2$ \\
\hline 219.0300 & 219.0300 & 0.47 & 8.5 & $\mathrm{C} 11 \mathrm{H} 7 \mathrm{O} 5$ \\
\hline 219.0510 & 219.0510 & 0.00 & 3.5 & $\mathrm{C} 8 \mathrm{H} 11 \mathrm{O} 7$ \\
\hline 219.0665 & 219.0663 & 0.81 & 7.5 & $\mathrm{C} 12 \mathrm{H} 11 \mathrm{O} 4$ \\
\hline 219.1028 & 219.1027 & 0.63 & 6.5 & $\mathrm{C} 13 \mathrm{H} 15 \mathrm{O} 3$ \\
\hline 219.1392 & 219.1391 & 0.47 & 5.5 & $\mathrm{C} 14 \mathrm{H} 19 \mathrm{O} 2$ \\
\hline 221.0457 & 221.0456 & 0.83 & 7.5 & C11 H9 O5 \\
\hline 221.0668 & 221.0668 & 0.43 & 2.5 & $\mathrm{C} 8 \mathrm{H} 13 \mathrm{O} 7$ \\
\hline 221.0821 & 221.0819 & 0.72 & 6.5 & $\mathrm{C} 12 \mathrm{H} 13 \mathrm{O} 4$ \\
\hline 221.1183 & 221.1183 & -0.08 & 5.5 & $\mathrm{C} 13 \mathrm{H} 17 \mathrm{O} 3$ \\
\hline 221.1549 & 221.1547 & 0.99 & 4.5 & $\mathrm{C} 14 \mathrm{H} 21 \mathrm{O} 2$ \\
\hline 223.0248 & 223.0248 & -0.06 & 7.5 & C10 H7 O6 \\
\hline 223.0403 & 223.0401 & 0.82 & 11.5 & $\mathrm{C} 14 \mathrm{H} 7 \mathrm{O} 3$ \\
\hline 223.0614 & 223.0612 & 0.89 & 6.5 & $\mathrm{C} 11 \mathrm{H} 11 \mathrm{O} 5$ \\
\hline 223.0766 & 223.0765 & 0.53 & 10.5 & $\mathrm{C} 15 \mathrm{H} 11 \mathrm{O} 2$ \\
\hline 223.0825 & 223.0824 & 0.56 & 1.5 & C8 H15 O7 \\
\hline 223.0978 & 223.0976 & 1.02 & 5.5 & $\mathrm{C} 12 \mathrm{H} 15 \mathrm{O} 4$ \\
\hline 223.1342 & 223.1340 & 0.80 & 4.5 & $\mathrm{C} 13 \mathrm{H} 19 \mathrm{O} 3$ \\
\hline 223.1705 & 223.1704 & 0.71 & 3.5 & $\mathrm{C} 14 \mathrm{H} 23 \mathrm{O} 2$ \\
\hline 225.0405 & 225.0405 & 0.13 & 6.5 & C10 H9 O6 \\
\hline 225.0558 & 225.0558 & 0.28 & 10.5 & $\mathrm{C} 14 \mathrm{H} 9 \mathrm{O} 3$ \\
\hline 225.0771 & 225.0769 & 0.99 & 5.5 & $\mathrm{C} 11 \mathrm{H} 13 \mathrm{O} 5$ \\
\hline 225.0922 & 225.0922 & 0.21 & 9.5 & $\mathrm{C} 15 \mathrm{H} 13 \mathrm{O} 2$ \\
\hline 225.1134 & 225.1132 & 0.66 & 4.5 & $\mathrm{C} 12 \mathrm{H} 17 \mathrm{O} 4$ \\
\hline 225.1496 & 225.1496 & 0.07 & 3.5 & $\mathrm{C} 13 \mathrm{H} 21 \mathrm{O} 3$ \\
\hline 227.0350 & 227.0350 & 0.12 & 10.5 & $\mathrm{C} 13 \mathrm{H} 7 \mathrm{O} 4$ \\
\hline 227.0563 & 227.0565 & 0.79 & 5.5 & C10 H11 O6 \\
\hline 227.0716 & 227.0714 & 1.07 & 9.5 & $\mathrm{C} 14 \mathrm{H} 11 \mathrm{O} 3$ \\
\hline 227.0928 & 227.0925 & 1.12 & 4.5 & $\mathrm{C} 11 \mathrm{H} 15 \mathrm{O} 5$ \\
\hline 227.1078 & 227.1078 & 0.30 & 8.5 & $\mathrm{C} 15 \mathrm{H} 15 \mathrm{O} 2$ \\
\hline 227.1289 & 227.1289 & 0.06 & 3.5 & $\mathrm{C} 12 \mathrm{H} 19 \mathrm{O} 4$ \\
\hline 229.0507 & 229.0507 & 0.30 & 9.5 & C13 H9 O4 \\
\hline
\end{tabular}




\begin{tabular}{|c|c|c|c|c|}
\hline 229.0719 & 229.0718 & 0.53 & 4.5 & C10 H13 O6 \\
\hline 229.0872 & 229.0871 & 0.93 & 8.5 & $\mathrm{C} 14 \mathrm{H} 13 \mathrm{O} 3$ \\
\hline 229.1082 & 229.1082 & 0.12 & 3.5 & $\mathrm{C} 11 \mathrm{H} 17 \mathrm{O} 5$ \\
\hline 229.1236 & 229.1234 & 0.64 & 7.5 & $\mathrm{C} 15 \mathrm{H} 17 \mathrm{O} 2$ \\
\hline 231.0300 & 231.0300 & 0.27 & 9.5 & $\mathrm{C} 12 \mathrm{H} 7 \mathrm{O} 5$ \\
\hline 231.0514 & 231.0514 & 1.75 & 4.5 & C9 H11 O7 \\
\hline 231.0665 & 231.0663 & 0.94 & 8.5 & $\mathrm{C} 13 \mathrm{H} 11 \mathrm{O} 4$ \\
\hline 231.0876 & 231.0874 & 0.64 & 3.5 & C10 H15 O6 \\
\hline 231.1030 & 231.1027 & 1.29 & 7.5 & C14 H15 O3 \\
\hline 231.1240 & 231.1238 & 0.90 & 2.5 & C11 H19 O5 \\
\hline 231.1391 & 231.1391 & 0.27 & 6.5 & $\mathrm{C} 15 \mathrm{H} 19 \mathrm{O} 2$ \\
\hline 233.0457 & 233.0456 & 0.74 & 8.5 & $\mathrm{C} 12 \mathrm{H} 9 \mathrm{O} 5$ \\
\hline 233.0669 & 233.0670 & 1.00 & 3.5 & $\mathrm{C} 9 \mathrm{H} 13 \mathrm{O} 7$ \\
\hline 233.0821 & 233.0820 & 0.81 & 7.5 & $\mathrm{C} 13 \mathrm{H} 13 \mathrm{O} 4$ \\
\hline 233.1034 & 233.1034 & 1.28 & 2.5 & C10 H17 O6 \\
\hline 233.1184 & 233.1183 & 0.22 & 6.5 & $\mathrm{C} 14 \mathrm{H} 17 \mathrm{O} 3$ \\
\hline 233.1550 & 233.1547 & 1.17 & 5.5 & $\mathrm{C} 15 \mathrm{H} 21 \mathrm{O} 2$ \\
\hline 235.0250 & 235.0252 & 0.93 & 8.5 & C11 H7 O6 \\
\hline 235.0614 & 235.0613 & 0.91 & 7.5 & C12 H11 O5 \\
\hline 235.0824 & 235.0824 & 0.40 & 2.5 & C9 H15 O7 \\
\hline 235.0979 & 235.0976 & 1.30 & 6.5 & $\mathrm{C} 13 \mathrm{H} 15 \mathrm{O} 4$ \\
\hline 235.1340 & 235.1340 & 0.21 & 5.5 & C14 H19 O3 \\
\hline 235.1705 & 235.1704 & 0.68 & 4.5 & $\mathrm{C} 15 \mathrm{H} 23 \mathrm{O} 2$ \\
\hline 237.0406 & 237.0405 & 0.54 & 7.5 & C11 H9 O6 \\
\hline 237.0618 & 237.0617 & 0.93 & 2.5 & C8 H13 O8 \\
\hline 237.0771 & 237.0769 & 1.09 & 6.5 & $\mathrm{C} 12 \mathrm{H} 13 \mathrm{O} 5$ \\
\hline 237.0922 & 237.0922 & 0.54 & 10.5 & $\mathrm{C} 16 \mathrm{H} 13 \mathrm{O} 2$ \\
\hline 237.1133 & 237.1132 & 0.15 & 5.5 & C13 H17 O4 \\
\hline 237.1497 & 237.1496 & 0.19 & 4.5 & $\mathrm{C} 14 \mathrm{H} 21 \mathrm{O} 3$ \\
\hline 239.0561 & 239.0561 & -0.09 & 6.5 & C11 H11 O6 \\
\hline 239.0716 & 239.0714 & 0.81 & 10.5 & C15 H11 O3 \\
\hline 239.0927 & 239.0925 & 0.74 & 5.5 & $\mathrm{C} 12 \mathrm{H} 15 \mathrm{O} 5$ \\
\hline 239.1078 & 239.1078 & 0.32 & 9.5 & $\mathrm{C} 16 \mathrm{H} 15 \mathrm{O} 2$ \\
\hline 239.1291 & 239.1289 & 0.76 & 4.5 & $\mathrm{C} 13 \mathrm{H} 19 \mathrm{O} 4$ \\
\hline 239.1655 & 239.1653 & 0.89 & 3.5 & $\mathrm{C} 14 \mathrm{H} 23 \mathrm{O} 3$ \\
\hline 241.0508 & 241.0507 & 0.86 & 10.5 & C14 H9 O4 \\
\hline 241.0718 & 241.0718 & 0.23 & 5.5 & C11 H13 O6 \\
\hline 241.0873 & 241.0871 & 1.09 & 9.5 & C15 H13 O3 \\
\hline 241.1234 & 241.1234 & -0.14 & 8.5 & $\mathrm{C} 16 \mathrm{H} 17 \mathrm{O} 2$ \\
\hline 241.1447 & 241.1445 & 0.71 & 3.5 & $\mathrm{C} 13 \mathrm{H} 21 \mathrm{O} 4$ \\
\hline 241.1810 & 241.1809 & 0.33 & 2.5 & $\mathrm{C} 14 \mathrm{H} 25 \mathrm{O} 3$ \\
\hline 241.2176 & 241.2173 & 1.11 & 1.5 & $\mathrm{C} 15 \mathrm{H} 29 \mathrm{O} 2$ \\
\hline 243.0514 & 243.0514 & 1.46 & 5.5 & C10 H11 O7 \\
\hline
\end{tabular}




\begin{tabular}{|c|c|c|c|c|}
\hline 243.0666 & 243.0663 & 1.43 & 9.5 & C14 H11 O4 \\
\hline 243.0878 & 243.0878 & 1.52 & 4.5 & $\mathrm{C} 11 \mathrm{H} 15 \mathrm{O} 6$ \\
\hline 243.1030 & 243.1027 & 1.33 & 8.5 & $\mathrm{C} 15 \mathrm{H} 15 \mathrm{O} 3$ \\
\hline 243.1240 & 243.1238 & 0.79 & 3.5 & $\mathrm{C} 12 \mathrm{H} 19 \mathrm{O5}$ \\
\hline 243.1394 & 243.1391 & 1.60 & 7.5 & $\mathrm{C} 16 \mathrm{H} 19 \mathrm{O} 2$ \\
\hline 245.0457 & 245.0456 & 0.67 & 9.5 & C13 H9 O5 \\
\hline 245.0669 & 245.0668 & 0.71 & 4.5 & $\mathrm{C} 10 \mathrm{H} 13 \mathrm{O} 7$ \\
\hline 245.0822 & 245.0820 & 1.30 & 8.5 & $\mathrm{C} 14 \mathrm{H} 13 \mathrm{O} 4$ \\
\hline 245.1029 & 245.1031 & -0.77 & 3.5 & $\mathrm{C} 11 \mathrm{H} 17 \mathrm{O} 6$ \\
\hline 245.1185 & 245.1184 & 0.58 & 7.5 & $\mathrm{C} 15 \mathrm{H} 17 \mathrm{O} 3$ \\
\hline 247.0615 & 247.0613 & 0.94 & 8.5 & C13 H11 O5 \\
\hline 247.0827 & 247.0827 & 1.51 & 3.5 & $\mathrm{C} 10 \mathrm{H} 15 \mathrm{O} 7$ \\
\hline 247.0979 & 247.0976 & 1.35 & 7.5 & $\mathrm{C} 14 \mathrm{H} 15 \mathrm{O} 4$ \\
\hline 247.1343 & 247.1340 & 1.16 & 6.5 & $\mathrm{C} 15 \mathrm{H} 19 \mathrm{O} 3$ \\
\hline 247.1706 & 247.1704 & 0.87 & 5.5 & $\mathrm{C} 16 \mathrm{H} 23 \mathrm{O} 2$ \\
\hline 249.0407 & 249.0405 & 0.84 & 8.5 & $\mathrm{C} 12 \mathrm{H} 9 \mathrm{O} 6$ \\
\hline 249.0619 & 249.0619 & 1.32 & 3.5 & C9 H13 O8 \\
\hline 249.0772 & 249.0769 & 1.30 & 7.5 & $\mathrm{C} 13 \mathrm{H} 13 \mathrm{O} 5$ \\
\hline 249.0983 & 249.0983 & 1.18 & 2.5 & $\mathrm{C} 10 \mathrm{H} 17 \mathrm{O} 7$ \\
\hline 249.1134 & 249.1132 & 0.72 & 6.5 & $\mathrm{C} 14 \mathrm{H} 17 \mathrm{O} 4$ \\
\hline 249.1499 & 249.1496 & 1.28 & 5.5 & $\mathrm{C} 15 \mathrm{H} 21 \mathrm{O} 3$ \\
\hline 251.0564 & 251.0565 & 1.07 & 7.5 & $\mathrm{C} 12 \mathrm{H} 11 \mathrm{O} 6$ \\
\hline 251.0722 & 251.0723 & 3.20 & 11.5 & $\mathrm{C} 16 \mathrm{H} 11 \mathrm{O} 3$ \\
\hline 251.0776 & 251.0776 & 1.47 & 2.5 & C9 H15 O8 \\
\hline 251.0929 & 251.0925 & 1.66 & 6.5 & $\mathrm{C} 13 \mathrm{H} 15 \mathrm{O} 5$ \\
\hline 251.1082 & 251.1078 & 1.82 & 10.5 & $\mathrm{C} 17 \mathrm{H} 15 \mathrm{O} 2$ \\
\hline 251.1293 & 251.1289 & 1.45 & 5.5 & $\mathrm{C} 14 \mathrm{H} 19 \mathrm{O} 4$ \\
\hline 251.1654 & 251.1653 & 0.50 & 4.5 & $\mathrm{C} 15 \mathrm{H} 23 \mathrm{O} 3$ \\
\hline 253.0356 & 253.0355 & 0.85 & 7.5 & C11 H9 O7 \\
\hline 253.0509 & 253.0507 & 1.22 & 11.5 & $\mathrm{C} 15 \mathrm{H} 9 \mathrm{O} 4$ \\
\hline 253.0720 & 253.0721 & 0.94 & 6.5 & $\mathrm{C} 12 \mathrm{H} 13 \mathrm{O} 6$ \\
\hline 253.0873 & 253.0871 & 1.16 & 10.5 & $\mathrm{C} 16 \mathrm{H} 13 \mathrm{O} 3$ \\
\hline 253.1083 & 253.1082 & 0.75 & 5.5 & C13 H17 O5 \\
\hline 253.1241 & 253.1243 & 2.75 & 9.5 & $\mathrm{C} 17 \mathrm{H} 17 \mathrm{O} 2$ \\
\hline 253.1448 & 253.1445 & 1.00 & 4.5 & $\mathrm{C} 14 \mathrm{H} 21 \mathrm{O} 4$ \\
\hline 253.1814 & 253.1809 & 1.82 & 3.5 & $\mathrm{C} 15 \mathrm{H} 25 \mathrm{O} 3$ \\
\hline 253.2175 & 253.2173 & 0.79 & 2.5 & $\mathrm{C} 16 \mathrm{H} 29 \mathrm{O} 2$ \\
\hline 255.0665 & 255.0663 & 0.97 & 10.5 & $\mathrm{C} 15 \mathrm{H} 11 \mathrm{O} 4$ \\
\hline 255.0876 & 255.0878 & 0.70 & 5.5 & $\mathrm{C} 12 \mathrm{H} 15 \mathrm{O} 6$ \\
\hline 255.1029 & 255.1027 & 0.95 & 9.5 & $\mathrm{C} 16 \mathrm{H} 15 \mathrm{O} 3$ \\
\hline 255.1240 & 255.1238 & 0.78 & 4.5 & C13 H19 O5 \\
\hline 255.1604 & 255.1602 & 1.02 & 3.5 & $\mathrm{C} 14 \mathrm{H} 23 \mathrm{O} 4$ \\
\hline 257.0456 & 257.0456 & 0.09 & 10.5 & $\mathrm{C} 14 \mathrm{H} 9 \mathrm{O} 5$ \\
\hline
\end{tabular}




\begin{tabular}{|c|c|c|c|c|}
\hline 257.0670 & 257.0670 & 1.42 & 5.5 & C11 H13 O7 \\
\hline 257.0822 & 257.0820 & 1.12 & 9.5 & $\mathrm{C} 15 \mathrm{H} 13 \mathrm{O} 4$ \\
\hline 257.1031 & 257.1031 & 0.15 & 4.5 & C12 H17 O6 \\
\hline 257.1186 & 257.1184 & 1.06 & 8.5 & $\mathrm{C} 16 \mathrm{H} 17 \mathrm{O} 3$ \\
\hline 257.1397 & 257.1395 & 0.94 & 3.5 & $\mathrm{C} 13 \mathrm{H} 21 \mathrm{O} 5$ \\
\hline 259.0616 & 259.0613 & 1.52 & 9.5 & C14 H11 O5 \\
\hline 259.0827 & 259.0827 & 1.56 & 4.5 & $\mathrm{C} 11 \mathrm{H} 15 \mathrm{O} 7$ \\
\hline 259.0980 & 259.0976 & 1.81 & 8.5 & $\mathrm{C} 15 \mathrm{H} 15 \mathrm{O} 4$ \\
\hline 259.1343 & 259.1340 & 1.36 & 7.5 & C16 H19 O3 \\
\hline 261.0409 & 261.0408 & 1.80 & 9.5 & C13 H9 O6 \\
\hline 261.0618 & 261.0619 & 0.96 & 4.5 & $\mathrm{C} 10 \mathrm{H} 13 \mathrm{O} 8$ \\
\hline 261.0773 & 261.0769 & 1.54 & 8.5 & C14 H13 O5 \\
\hline 261.0984 & 261.0983 & 1.55 & 3.5 & $\mathrm{C} 11 \mathrm{H} 17 \mathrm{O} 7$ \\
\hline 261.1137 & 261.1133 & 1.70 & 7.5 & $\mathrm{C} 15 \mathrm{H} 17 \mathrm{O} 4$ \\
\hline 261.1500 & 261.1496 & 1.36 & 6.5 & $\mathrm{C} 16 \mathrm{H} 21 \mathrm{O} 3$ \\
\hline 263.0564 & 263.0565 & 1.10 & 8.5 & C13 H11 O6 \\
\hline 263.0778 & 263.0776 & 2.05 & 3.5 & $\mathrm{C} 10 \mathrm{H} 15 \mathrm{O} 8$ \\
\hline 263.0930 & 263.0934 & 1.91 & 7.5 & C14 H15 O5 \\
\hline 263.1291 & 263.1289 & 0.71 & 6.5 & C15 H19 O4 \\
\hline 263.1658 & 263.1653 & 1.99 & 5.5 & $\mathrm{C} 16 \mathrm{H} 23 \mathrm{O} 3$ \\
\hline 265.0356 & 265.0357 & 0.88 & 8.5 & $\mathrm{C} 12 \mathrm{H} 9 \mathrm{O} 7$ \\
\hline 265.0722 & 265.0721 & 1.73 & 7.5 & C13 H13 O6 \\
\hline 265.0874 & 265.0871 & 1.44 & 11.5 & $\mathrm{C} 17 \mathrm{H} 13 \mathrm{O} 3$ \\
\hline 265.0930 & 265.0930 & 0.41 & 2.5 & C10 H17 O8 \\
\hline 265.1086 & 265.1082 & 1.53 & 6.5 & C14 H17 O5 \\
\hline 265.1815 & 265.1809 & 2.21 & 4.5 & $\mathrm{C} 16 \mathrm{H} 25 \mathrm{O} 3$ \\
\hline 267.0513 & 267.0514 & 1.06 & 7.5 & C12 H11 O7 \\
\hline 267.0667 & 267.0663 & 1.64 & 11.5 & C16 H11 O4 \\
\hline 267.0878 & 267.0878 & 1.61 & 6.5 & C13 H15 O6 \\
\hline 267.1030 & 267.1027 & 1.06 & 10.5 & C17 H15 O3 \\
\hline 267.1242 & 267.1238 & 1.54 & 5.5 & C14 H19 O5 \\
\hline 267.1440 & 267.1437 & -3.39 & 0.5 & $\mathrm{C} 11 \mathrm{H} 23 \mathrm{O} 7$ \\
\hline 267.1608 & 267.1602 & 2.39 & 4.5 & $\mathrm{C} 15 \mathrm{H} 23 \mathrm{O} 4$ \\
\hline 267.1968 & 267.1966 & 0.88 & 3.5 & $\mathrm{C} 16 \mathrm{H} 27 \mathrm{O} 3$ \\
\hline 269.0670 & 269.0670 & 1.32 & 6.5 & $\mathrm{C} 12 \mathrm{H} 13 \mathrm{O} 7$ \\
\hline 269.0823 & 269.0820 & 1.48 & 10.5 & C16 H13 O4 \\
\hline 269.1032 & 269.1031 & 0.40 & 5.5 & C13 H17 O6 \\
\hline 269.1188 & 269.1184 & 1.72 & 9.5 & $\mathrm{C} 17 \mathrm{H} 17 \mathrm{O} 3$ \\
\hline 269.1399 & 269.1395 & 1.59 & 4.5 & $\mathrm{C} 14 \mathrm{H} 21 \mathrm{O} 5$ \\
\hline 269.1765 & 269.1758 & 2.35 & 3.5 & $\mathrm{C} 15 \mathrm{H} 25 \mathrm{O} 4$ \\
\hline 269.2490 & 269.2486 & 1.60 & 1.5 & $\mathrm{C} 17 \mathrm{H} 33 \mathrm{O} 2$ \\
\hline 271.0615 & 271.0613 & 1.01 & 10.5 & C15 H11 O5 \\
\hline 271.0826 & 271.0827 & 0.94 & 5.5 & $\mathrm{C} 12 \mathrm{H} 15 \mathrm{O} 7$ \\
\hline
\end{tabular}




\begin{tabular}{|c|c|c|c|c|}
\hline 271.0981 & 271.0985 & 1.91 & 9.5 & C16 H15 O4 \\
\hline 271.1345 & 271.1340 & 1.82 & 8.5 & C17 H19 O3 \\
\hline 271.2283 & 271.2279 & 1.74 & 1.5 & C16 H31 O3 \\
\hline 273.0774 & 273.0777 & 1.99 & 9.5 & $\mathrm{C} 15 \mathrm{H} 13 \mathrm{O} 5$ \\
\hline 273.1138 & 273.1133 & 2.01 & 8.5 & $\mathrm{C} 16 \mathrm{H} 17 \mathrm{O} 4$ \\
\hline 275.0566 & 275.0565 & 1.63 & 9.5 & C14 H11 O6 \\
\hline 275.0778 & 275.0776 & 2.03 & 4.5 & C11 H15 O8 \\
\hline 275.0930 & 275.0934 & 1.83 & 8.5 & $\mathrm{C} 15 \mathrm{H} 15 \mathrm{O} 5$ \\
\hline 275.1293 & 275.1289 & 1.59 & 7.5 & $\mathrm{C} 16 \mathrm{H} 19 \mathrm{O} 4$ \\
\hline 275.2020 & 275.2017 & 1.15 & 5.5 & $\mathrm{C} 18 \mathrm{H} 27 \mathrm{O} 2$ \\
\hline 277.0725 & 277.0726 & 2.49 & 8.5 & C14 H13 O6 \\
\hline 277.0931 & 277.0930 & 0.76 & 3.5 & $\mathrm{C} 11 \mathrm{H} 17 \mathrm{O} 8$ \\
\hline 277.1085 & 277.1082 & 1.01 & 7.5 & C15 H17 O5 \\
\hline 277.1452 & 277.1445 & 2.26 & 6.5 & $\mathrm{C} 16 \mathrm{H} 21 \mathrm{O} 4$ \\
\hline 279.0515 & 279.0514 & 1.59 & 8.5 & C13 H11 O7 \\
\hline 279.0878 & 279.0878 & 1.46 & 7.5 & C14 H15 O6 \\
\hline 279.1034 & 279.1036 & 2.59 & 11.5 & C18 H15 O3 \\
\hline 279.1090 & 279.1089 & 1.72 & 2.5 & C11 H19 O8 \\
\hline 279.1241 & 279.1238 & 0.97 & 6.5 & C15 H19 O5 \\
\hline 279.2333 & 279.2330 & 1.28 & 3.5 & $\mathrm{C} 18 \mathrm{H} 31 \mathrm{O} 2$ \\
\hline 281.0670 & 281.0670 & 1.08 & 7.5 & $\mathrm{C} 13 \mathrm{H} 13 \mathrm{O} 7$ \\
\hline 281.0824 & 281.0820 & 1.59 & 11.5 & $\mathrm{C} 17 \mathrm{H} 13 \mathrm{O} 4$ \\
\hline 281.0880 & 281.0879 & 0.59 & 2.5 & $\mathrm{C} 10 \mathrm{H} 17 \mathrm{O} 9$ \\
\hline 281.1034 & 281.1034 & 1.35 & 6.5 & C14 H17 O6 \\
\hline 281.1188 & 281.1184 & 1.54 & 10.5 & $\mathrm{C} 18 \mathrm{H} 17 \mathrm{O} 3$ \\
\hline 281.2490 & 281.2486 & 1.56 & 2.5 & $\mathrm{C} 18 \mathrm{H} 33 \mathrm{O} 2$ \\
\hline 283.0617 & 283.0621 & 1.81 & 11.5 & C16 H11 O5 \\
\hline 283.0828 & 283.0827 & 1.75 & 6.5 & $\mathrm{C} 13 \mathrm{H} 15 \mathrm{O} 7$ \\
\hline 283.0981 & 283.0976 & 1.65 & 10.5 & $\mathrm{C} 17 \mathrm{H} 15 \mathrm{O} 4$ \\
\hline 283.1345 & 283.1349 & 1.95 & 9.5 & C18 H19 O3 \\
\hline 283.1920 & 283.1915 & 1.80 & 3.5 & $\mathrm{C} 16 \mathrm{H} 27 \mathrm{O} 4$ \\
\hline 285.0774 & 285.0777 & 1.77 & 10.5 & C16 H13 O5 \\
\hline 285.1137 & 285.1133 & 1.54 & 9.5 & C17 H17 O4 \\
\hline 287.0567 & 287.0565 & 2.02 & 10.5 & C15 H11 O6 \\
\hline 287.0931 & 287.0934 & 2.00 & 9.5 & $\mathrm{C} 16 \mathrm{H} 15 \mathrm{O} 5$ \\
\hline 287.1294 & 287.1289 & 1.63 & 8.5 & C17 H19 O4 \\
\hline 287.2232 & 287.2228 & 1.39 & 1.5 & C16 H31 O4 \\
\hline 289.0722 & 289.0721 & 1.48 & 9.5 & C15 H13 O6 \\
\hline 289.0934 & 289.0932 & 1.76 & 4.5 & C12 H17 O8 \\
\hline 289.1087 & 289.1090 & 1.91 & 8.5 & C16 H17 O5 \\
\hline 289.1454 & 289.1459 & 3.00 & 7.5 & $\mathrm{C} 17 \mathrm{H} 21 \mathrm{O} 4$ \\
\hline 289.1818 & 289.1825 & 2.91 & 6.5 & $\mathrm{C} 18 \mathrm{H} 25 \mathrm{O} 3$ \\
\hline 291.0881 & 291.0883 & 2.13 & 8.5 & C15 H15 O6 \\
\hline
\end{tabular}




\begin{tabular}{|c|c|c|c|c|}
\hline 291.1242 & 291.1238 & 1.25 & 7.5 & C16 H19 O5 \\
\hline 291.1605 & 291.1602 & 1.22 & 6.5 & $\mathrm{C} 17 \mathrm{H} 23 \mathrm{O} 4$ \\
\hline 291.1971 & 291.1966 & 1.74 & 5.5 & $\mathrm{C} 18 \mathrm{H} 27 \mathrm{O} 3$ \\
\hline 293.0673 & 293.0670 & 2.06 & 8.5 & $\mathrm{C} 14 \mathrm{H} 13 \mathrm{O} 7$ \\
\hline 293.0883 & 293.0882 & 1.62 & 3.5 & C11 H17 O9 \\
\hline 293.1035 & 293.1034 & 1.63 & 7.5 & C15 H17 O6 \\
\hline 293.1763 & 293.1758 & 1.55 & 5.5 & $\mathrm{C} 17 \mathrm{H} 25 \mathrm{O} 4$ \\
\hline 293.2128 & 293.2122 & 2.14 & 4.5 & $\mathrm{C} 18 \mathrm{H} 29 \mathrm{O} 3$ \\
\hline 295.0829 & 295.0827 & 1.81 & 7.5 & C14 H15 O7 \\
\hline 295.0981 & 295.0985 & 1.76 & 11.5 & C18 H15 O4 \\
\hline 295.1193 & 295.1191 & 1.86 & 6.5 & C15 H19 O6 \\
\hline 295.1555 & 295.1551 & 1.46 & 5.5 & $\mathrm{C} 16 \mathrm{H} 23 \mathrm{O} 5$ \\
\hline 295.2283 & 295.2279 & 1.31 & 3.5 & $\mathrm{C} 18 \mathrm{H} 31 \mathrm{O} 3$ \\
\hline 297.0773 & 297.0777 & 1.63 & 11.5 & $\mathrm{C} 17 \mathrm{H} 13 \mathrm{O} 5$ \\
\hline 297.0984 & 297.0983 & 1.56 & 6.5 & $\mathrm{C} 14 \mathrm{H} 17 \mathrm{O} 7$ \\
\hline 297.1138 & 297.1142 & 1.94 & 10.5 & $\mathrm{C} 18 \mathrm{H} 17 \mathrm{O} 4$ \\
\hline 297.1713 & 297.1716 & 1.79 & 4.5 & $\mathrm{C} 16 \mathrm{H} 25 \mathrm{O} 5$ \\
\hline 297.2441 & 297.2435 & 1.91 & 2.5 & $\mathrm{C} 18 \mathrm{H} 33 \mathrm{O} 3$ \\
\hline 299.0564 & 299.0565 & 0.90 & 11.5 & C16 H11 O6 \\
\hline 299.0929 & 299.0926 & 1.38 & 10.5 & $\mathrm{C} 17 \mathrm{H} 15 \mathrm{O} 5$ \\
\hline 299.1295 & 299.1298 & 1.96 & 9.5 & $\mathrm{C} 18 \mathrm{H} 19 \mathrm{O} 4$ \\
\hline 299.2595 & 299.2592 & 1.13 & 1.5 & $\mathrm{C} 18 \mathrm{H} 35 \mathrm{O} 3$ \\
\hline 301.0723 & 301.0721 & 1.69 & 10.5 & C16 H13 O6 \\
\hline 301.1087 & 301.1090 & 1.97 & 9.5 & C17 H17 O5 \\
\hline 301.1451 & 301.1446 & 2.02 & 8.5 & $\mathrm{C} 18 \mathrm{H} 21 \mathrm{O} 4$ \\
\hline 303.0880 & 303.0878 & 1.78 & 9.5 & C16 H15 O6 \\
\hline 303.1243 & 303.1247 & 1.69 & 8.5 & C17 H19 O5 \\
\hline 305.0670 & 305.0670 & 1.10 & 9.5 & C15 H13 O7 \\
\hline 305.0882 & 305.0882 & 1.39 & 4.5 & C12 H17 O9 \\
\hline 305.1036 & 305.1034 & 1.80 & 8.5 & C16 H17 O6 \\
\hline 305.1403 & 305.1403 & 2.73 & 7.5 & $\mathrm{C} 17 \mathrm{H} 21 \mathrm{O} 5$ \\
\hline 307.0829 & 307.0827 & 1.90 & 8.5 & $\mathrm{C} 15 \mathrm{H} 15 \mathrm{O} 7$ \\
\hline 307.1040 & 307.1038 & 1.68 & 3.5 & C12 H19 O9 \\
\hline 307.1191 & 307.1191 & 1.23 & 7.5 & C16 H19 O6 \\
\hline 307.1924 & 307.1928 & 2.99 & 5.5 & $\mathrm{C} 18 \mathrm{H} 27 \mathrm{O} 4$ \\
\hline 309.0987 & 309.0988 & 2.18 & 7.5 & C15 H17 O7 \\
\hline 309.1718 & 309.1716 & 3.28 & 5.5 & $\mathrm{C} 17 \mathrm{H} 25 \mathrm{O} 5$ \\
\hline 309.2082 & 309.2085 & 3.45 & 4.5 & $\mathrm{C} 18 \mathrm{H} 29 \mathrm{O} 4$ \\
\hline 311.0931 & 311.0934 & 1.78 & 11.5 & $\mathrm{C} 18 \mathrm{H} 15 \mathrm{O} 5$ \\
\hline 311.1143 & 311.1145 & 2.01 & 6.5 & C15 H19 O7 \\
\hline 311.1297 & 311.1298 & 2.50 & 10.5 & C19 H19 O4 \\
\hline 311.2234 & 311.2228 & 1.93 & 3.5 & $\mathrm{C} 18 \mathrm{H} 31 \mathrm{O} 4$ \\
\hline 313.0724 & 313.0726 & 2.07 & 11.5 & C17 H13 O6 \\
\hline
\end{tabular}




\begin{tabular}{|c|c|c|c|c|c|}
\hline & 313.0933 & 313.0932 & 1.15 & 6.5 & $\mathrm{C} 14 \mathrm{H} 17 \mathrm{O} 8$ \\
\hline & 313.1089 & 313.1090 & 2.34 & 10.5 & C18 H17 O5 \\
\hline & 313.2387 & 313.2384 & 0.81 & 2.5 & C18 H33 O4 \\
\hline & 315.0881 & 315.0883 & 2.09 & 10.5 & C17 H15 O6 \\
\hline & 315.1244 & 315.1247 & 1.98 & 9.5 & C18 H19 O5 \\
\hline & 317.0673 & 317.0675 & 2.09 & 10.5 & C16 H13 O7 \\
\hline & 317.1036 & 317.1034 & 1.76 & 9.5 & C17 H17 O6 \\
\hline & 317.1401 & 317.1403 & 2.06 & 8.5 & $\mathrm{C} 18 \mathrm{H} 21 \mathrm{O} 5$ \\
\hline & 319.0828 & 319.0827 & 1.49 & 9.5 & C16 H15 O7 \\
\hline & 319.1193 & 319.1191 & 1.75 & 8.5 & C17 H19 O6 \\
\hline & 321.0988 & 321.0988 & 2.57 & 8.5 & $\mathrm{C} 16 \mathrm{H} 17 \mathrm{O} 7$ \\
\hline & 323.0991 & 323.0992 & 2.26 & 3.5 & C12 H19 O10 \\
\hline & 323.1141 & 323.1140 & 1.47 & 7.5 & C16 H19 O7 \\
\hline & 325.0935 & 325.0938 & 2.00 & 7.5 & $\mathrm{C} 15 \mathrm{H} 17 \mathrm{O} 8$ \\
\hline & 325.1088 & 325.1090 & 2.04 & 11.5 & C19 H17 O5 \\
\hline & 327.0882 & 327.0883 & 2.47 & 11.5 & C18 H15 O6 \\
\hline & 327.1245 & 327.1247 & 2.12 & 10.5 & C19 H19 O5 \\
\hline & 329.0674 & 329.0675 & 2.29 & 11.5 & $\mathrm{C} 17 \mathrm{H} 13 \mathrm{O} 7$ \\
\hline & 329.1038 & 329.1039 & 2.12 & 10.5 & C18 H17 O6 \\
\hline & 329.1403 & 329.1403 & 2.65 & 9.5 & $\mathrm{C} 19 \mathrm{H} 21 \mathrm{O} 5$ \\
\hline & 331.0831 & 331.0832 & 2.46 & 10.5 & C17 H15 O7 \\
\hline & 331.1196 & 331.1196 & 2.80 & 9.5 & C18 H19 O6 \\
\hline & 333.0989 & 333.0989 & 2.77 & 9.5 & $\mathrm{C} 17 \mathrm{H} 17 \mathrm{O} 7$ \\
\hline & 333.1353 & 333.1352 & 2.73 & 8.5 & $\mathrm{C} 18 \mathrm{H} 21 \mathrm{O} 6$ \\
\hline & 339.1244 & 339.1247 & 1.72 & 11.5 & C20 H19 O5 \\
\hline & 341.1040 & 341.1040 & 2.78 & 11.5 & C19 H17 O6 \\
\hline & 343.1195 & 343.1196 & 2.41 & 10.5 & C19 H19 O6 \\
\hline & 345.0989 & 345.0988 & 2.59 & 10.5 & C18 H17 O7 \\
\hline & 355.1194 & 355.1196 & 2.00 & 11.5 & C20 H19 O6 \\
\hline & 357.0627 & 357.0629 & 3.22 & 12.5 & C18 H13 O8 \\
\hline \multirow{13}{*}{ CHOS } & 91.0219 & 91.0218 & -4.16 & 0.5 & $\mathrm{C} 3 \mathrm{H} 7 \mathrm{O} \mathrm{S}$ \\
\hline & 93.0012 & 93.0011 & -4.23 & 0.5 & $\mathrm{C} 2 \mathrm{H} 5 \mathrm{O} 2 \mathrm{~S}$ \\
\hline & 108.9961 & 108.9960 & -0.03 & 0.5 & $\mathrm{C} 2 \mathrm{H} 5 \mathrm{O} 3 \mathrm{~S}$ \\
\hline & 110.9755 & 110.9752 & -2.46 & 0.5 & $\mathrm{C} \mathrm{H} 3 \mathrm{O} 4 \mathrm{~S}$ \\
\hline & 124.9548 & 124.9550 & -1.89 & 1.5 & $\mathrm{CHO} \mathrm{OS}$ \\
\hline & 132.9962 & 132.9960 & -2.54 & 2.5 & $\mathrm{C} 4 \mathrm{H} 5 \mathrm{O} 3 \mathrm{~S}$ \\
\hline & 134.9753 & 134.9752 & -3.65 & 2.5 & $\mathrm{C} 3 \mathrm{H} 3 \mathrm{O} 4 \mathrm{~S}$ \\
\hline & 138.9703 & 138.9702 & -2.57 & 1.5 & $\mathrm{C} 2 \mathrm{H} 3 \mathrm{O} 5 \mathrm{~S}$ \\
\hline & 138.9860 & 138.9859 & 0.44 & 5.5 & $\mathrm{C} 6 \mathrm{H} 3 \mathrm{O} 2 \mathrm{~S}$ \\
\hline & 151.0069 & 151.0071 & -1.05 & 1.5 & $\mathrm{C} 4 \mathrm{H} 7 \mathrm{O} 4 \mathrm{~S}$ \\
\hline & 152.9861 & 152.9863 & -1.41 & 1.5 & $\mathrm{C} 3 \mathrm{H} 5 \mathrm{O} 5 \mathrm{~S}$ \\
\hline & 169.0174 & 169.0176 & -1.07 & 0.5 & $\mathrm{C} 4 \mathrm{H} 9 \mathrm{O} 5 \mathrm{~S}$ \\
\hline & 170.9968 & 170.9969 & -0.78 & 0.5 & $\mathrm{C} 3 \mathrm{H} 7 \mathrm{O} 6 \mathrm{~S}$ \\
\hline
\end{tabular}




\begin{tabular}{|c|c|c|c|c|c|}
\hline & 175.0068 & 175.0071 & -1.34 & 3.5 & $\mathrm{C} 6 \mathrm{H} 7 \mathrm{O} 4 \mathrm{~S}$ \\
\hline & 188.9864 & 188.9863 & 0.62 & 4.5 & $\mathrm{C} 6 \mathrm{H} 5 \mathrm{O} 5 \mathrm{~S}$ \\
\hline & 191.0019 & 191.0020 & -0.47 & 3.5 & $\mathrm{C} 6 \mathrm{H} 7 \mathrm{O} 5 \mathrm{~S}$ \\
\hline & 193.0174 & 193.0176 & -0.90 & 2.5 & $\mathrm{C} 6 \mathrm{H} 9 \mathrm{O} 5 \mathrm{~S}$ \\
\hline & 194.9959 & 194.9957 & -4.93 & 2.5 & $\mathrm{C} 5 \mathrm{H} 7 \mathrm{O} 6 \mathrm{~S}$ \\
\hline & 203.0021 & 203.0020 & 0.47 & 4.5 & $\mathrm{C} 7 \mathrm{H} 7 \mathrm{O} 5 \mathrm{~S}$ \\
\hline & 205.0175 & 205.0176 & -0.80 & 3.5 & $\mathrm{C} 7 \mathrm{H} 9 \mathrm{O} 5 \mathrm{~S}$ \\
\hline & 209.0125 & 209.0125 & -0.32 & 2.5 & C6 H9 O6 S \\
\hline & 210.9921 & 210.9922 & 1.20 & 2.5 & $\mathrm{C} 5 \mathrm{H} 7 \mathrm{O} 7 \mathrm{~S}$ \\
\hline & 211.0281 & 211.0282 & -0.19 & 1.5 & C6 H11 O6 S \\
\hline & 217.0178 & 217.0176 & 0.92 & 4.5 & $\mathrm{C} 8 \mathrm{H} 9 \mathrm{O} 5 \mathrm{~S}$ \\
\hline & 217.0329 & 217.0329 & 0.01 & 8.5 & $\mathrm{C} 12 \mathrm{H} 9 \mathrm{O} 2 \mathrm{~S}$ \\
\hline & 221.0126 & 221.0125 & 0.25 & 3.5 & C7 H9 O6 S \\
\hline & 223.0283 & 223.0282 & 0.37 & 2.5 & C7 H11 O6 S \\
\hline & 228.9661 & 228.9661 & 0.54 & 2.5 & C4 H5 O9 S \\
\hline & 229.0329 & 229.0329 & 0.05 & 9.5 & $\mathrm{C} 13 \mathrm{H} 9 \mathrm{O} 2 \mathrm{~S}$ \\
\hline & 237.0579 & 237.0579 & -4.89 & 6.5 & $\mathrm{C} 12 \mathrm{H} 13 \mathrm{O} 3 \mathrm{~S}$ \\
\hline & 239.0231 & 239.0231 & 0.19 & 2.5 & C7 H11 O7 S \\
\hline & 241.0028 & 241.0027 & 1.99 & 2.5 & C6 H9 O8 S \\
\hline & 253.1118 & 253.1115 & 1.11 & 0.5 & $\mathrm{C} 10 \mathrm{H} 21 \mathrm{O} 5 \mathrm{~S}$ \\
\hline & 261.0077 & 261.0078 & 0.97 & 5.5 & C9 H9 O7 S \\
\hline & 261.1883 & 261.1889 & -4.15 & 0.5 & $\mathrm{C} 14 \mathrm{H} 29 \mathrm{O} 2 \mathrm{~S}$ \\
\hline & 268.9796 & 268.9795 & 0.19 & 3.5 & C7 H9 O7 S2 \\
\hline & 295.1592 & 295.1592 & 2.48 & 0.5 & $\mathrm{C} 13 \mathrm{H} 27 \mathrm{O} 5 \mathrm{~S}$ \\
\hline & 298.9903 & 298.9902 & 0.73 & 3.5 & C8 H11 O8 S2 \\
\hline & 317.2512 & 317.2515 & -2.41 & 0.5 & $\mathrm{C} 18 \mathrm{H} 37 \mathrm{O} 2 \mathrm{~S}$ \\
\hline & 351.2218 & 351.2218 & 2.17 & 0.5 & $\mathrm{C} 17 \mathrm{H} 35 \mathrm{O} 5 \mathrm{~S}$ \\
\hline & 94.0297 & 94.0298 & -1.57 & 4.5 & $\mathrm{C} 5 \mathrm{H} 4 \mathrm{ON}$ \\
\hline $\mathrm{CHON}$ & 102.0559 & 102.0561 & -1.19 & 1.5 & $\mathrm{C} 4 \mathrm{H} 8 \mathrm{O} 2 \mathrm{~N}$ \\
\hline & 108.0454 & 108.0455 & -1.06 & 4.5 & C6 H6 O N \\
\hline & 110.0610 & 110.0611 & -1.25 & 3.5 & C6 H8 O N \\
\hline & 118.0297 & 118.0298 & -1.09 & 6.5 & $\mathrm{C} 7 \mathrm{H} 4 \mathrm{ON}$ \\
\hline & 120.0454 & 120.0455 & -0.90 & 5.5 & C7 H6 O N \\
\hline & 122.0247 & 122.0248 & -0.74 & 5.5 & $\mathrm{C} 6 \mathrm{H} 4 \mathrm{O} 2 \mathrm{~N}$ \\
\hline & 122.0610 & 122.0611 & -0.78 & 4.5 & $\mathrm{C} 7 \mathrm{H} 8 \mathrm{O} N$ \\
\hline & 124.0767 & 124.0768 & -1.04 & 3.5 & C7 H10 O N \\
\hline & 128.0716 & 128.0717 & -0.59 & 2.5 & $\mathrm{C} 6 \mathrm{H} 10 \mathrm{O} 2 \mathrm{~N}$ \\
\hline & 130.0509 & 130.0510 & -0.53 & 2.5 & $\mathrm{C} 5 \mathrm{H} 8 \mathrm{O} 3 \mathrm{~N}$ \\
\hline & 132.0454 & 132.0455 & -0.63 & 6.5 & $\mathrm{C} 8 \mathrm{H} 6 \mathrm{O} \mathrm{N}$ \\
\hline & 132.0665 & 132.0666 & -0.60 & 1.5 & C5 H10 O3 N \\
\hline & 134.0247 & 134.0248 & -0.76 & 6.5 & $\mathrm{C} 7 \mathrm{H} 4 \mathrm{O} 2 \mathrm{~N}$ \\
\hline & 134.0611 & 134.0611 & -0.63 & 5.5 & $\mathrm{C} 8 \mathrm{H} 8 \mathrm{O} \mathrm{N}$ \\
\hline & 136.0404 & 136.0404 & -0.40 & 5.5 & $\mathrm{C} 7 \mathrm{H} 6 \mathrm{O} 2 \mathrm{~N}$ \\
\hline
\end{tabular}




\begin{tabular}{|c|c|c|c|c|}
\hline 136.0767 & 136.0768 & -0.54 & 4.5 & C8 H10 O N \\
\hline 137.0719 & 137.0720 & -0.76 & 4.5 & $\mathrm{C} 7 \mathrm{H} 9 \mathrm{O}$ N2 \\
\hline 138.0560 & 138.0561 & -0.55 & 4.5 & $\mathrm{C} 7 \mathrm{H} 8 \mathrm{O} 2 \mathrm{~N}$ \\
\hline 140.0716 & 140.0717 & -0.85 & 3.5 & $\mathrm{C} 7 \mathrm{H} 10 \mathrm{O} 2 \mathrm{~N}$ \\
\hline 142.0144 & 142.0146 & -1.62 & 4.5 & $\mathrm{C} 5 \mathrm{H} 4 \mathrm{O} 4 \mathrm{~N}$ \\
\hline 143.0250 & 143.0251 & -0.30 & 8.5 & $\mathrm{C} 8 \mathrm{H} 3 \mathrm{O}$ N2 \\
\hline 144.0454 & 144.0455 & -0.45 & 7.5 & C9 H6 O N \\
\hline 144.0666 & 144.0666 & -0.23 & 2.5 & C6 H10 O3 N \\
\hline 145.0406 & 145.0407 & -0.63 & 7.5 & C8 H5 O N2 \\
\hline 146.0247 & 146.0248 & -0.47 & 7.5 & $\mathrm{C} 8 \mathrm{H} 4 \mathrm{O} 2 \mathrm{~N}$ \\
\hline 146.0611 & 146.0611 & -0.17 & 6.5 & C9 H8 O N \\
\hline 147.0198 & 147.0200 & -1.09 & 7.5 & $\mathrm{C} 7 \mathrm{H} 3 \mathrm{O} 2 \mathrm{~N} 2$ \\
\hline 148.0404 & 148.0404 & -0.06 & 6.5 & $\mathrm{C} 8 \mathrm{H} 6 \mathrm{O} 2 \mathrm{~N}$ \\
\hline 148.0767 & 148.0768 & -0.50 & 5.5 & C9 H10 O N \\
\hline 150.0195 & 150.0197 & -0.96 & 6.5 & $\mathrm{C} 7 \mathrm{H} 4 \mathrm{O} 3 \mathrm{~N}$ \\
\hline 150.0560 & 150.0561 & -0.28 & 5.5 & $\mathrm{C} 8 \mathrm{H} 8 \mathrm{O} 2 \mathrm{~N}$ \\
\hline 150.0924 & 150.0924 & -0.24 & 4.5 & C9 H12 O N \\
\hline 151.0876 & 151.0877 & -0.33 & 4.5 & C8 H11 O N2 \\
\hline 152.0352 & 152.0353 & -0.73 & 5.5 & $\mathrm{C} 7 \mathrm{H} 6 \mathrm{O} 3 \mathrm{~N}$ \\
\hline 152.0717 & 152.0717 & -0.32 & 4.5 & $\mathrm{C} 8 \mathrm{H} 10 \mathrm{O} 2 \mathrm{~N}$ \\
\hline 153.0669 & 153.0670 & -0.30 & 4.5 & $\mathrm{C} 7 \mathrm{H} 9 \mathrm{O} 2 \mathrm{~N} 2$ \\
\hline 154.0509 & 154.0510 & -0.34 & 4.5 & $\mathrm{C} 7 \mathrm{H} 8 \mathrm{O} 3 \mathrm{~N}$ \\
\hline 154.0873 & 154.0874 & -0.34 & 3.5 & $\mathrm{C} 8 \mathrm{H} 12 \mathrm{O} 2 \mathrm{~N}$ \\
\hline 155.0826 & 155.0826 & -0.03 & 3.5 & C7 H11 O2 N2 \\
\hline 156.0302 & 156.0302 & -0.32 & 4.5 & C6 H6 O4 N \\
\hline 156.0666 & 156.0666 & -0.39 & 3.5 & $\mathrm{C} 7 \mathrm{H} 10 \mathrm{O} 3 \mathrm{~N}$ \\
\hline 158.0247 & 158.0248 & -0.23 & 8.5 & $\mathrm{C} 9 \mathrm{H} 4 \mathrm{O} 2 \mathrm{~N}$ \\
\hline 158.0458 & 158.0459 & -0.23 & 3.5 & C6 H8 O4 N \\
\hline 158.0611 & 158.0611 & -0.13 & 7.5 & $\mathrm{C} 10 \mathrm{H} 8 \mathrm{O} \mathrm{N}$ \\
\hline 158.0822 & 158.0823 & -0.22 & 2.5 & $\mathrm{C} 7 \mathrm{H} 12 \mathrm{O} 3 \mathrm{~N}$ \\
\hline 159.0564 & 159.0564 & -0.05 & 7.5 & C9 H7 O N2 \\
\hline 160.0404 & 160.0404 & 0.24 & 7.5 & $\mathrm{C} 9 \mathrm{H} 6 \mathrm{O} 2 \mathrm{~N}$ \\
\hline 160.0615 & 160.0615 & -0.41 & 2.5 & $\mathrm{C} 6 \mathrm{H} 10 \mathrm{O} 4 \mathrm{~N}$ \\
\hline 160.0768 & 160.0768 & -0.04 & 6.5 & $\mathrm{C} 10 \mathrm{H} 10 \mathrm{O} \mathrm{N}$ \\
\hline 161.0357 & 161.0357 & 0.02 & 7.5 & $\mathrm{C} 8 \mathrm{H} 5 \mathrm{O} 2 \mathrm{~N} 2$ \\
\hline 161.0720 & 161.0720 & -0.47 & 6.5 & C9 H9 O N2 \\
\hline 162.0196 & 162.0197 & -0.28 & 7.5 & $\mathrm{C} 8 \mathrm{H} 4 \mathrm{O} 3 \mathrm{~N}$ \\
\hline 162.0561 & 162.0561 & 0.07 & 6.5 & $\mathrm{C} 9 \mathrm{H} 8 \mathrm{O} 2 \mathrm{~N}$ \\
\hline 162.0924 & 162.0924 & -0.29 & 5.5 & $\mathrm{C} 10 \mathrm{H} 12 \mathrm{O} \mathrm{N}$ \\
\hline 163.0876 & 163.0877 & -0.47 & 5.5 & C9 H11 O N2 \\
\hline 164.0353 & 164.0353 & 0.00 & 6.5 & $\mathrm{C} 8 \mathrm{H} 6 \mathrm{O} 3 \mathrm{~N}$ \\
\hline 164.0717 & 164.0717 & -0.07 & 5.5 & $\mathrm{C} 9 \mathrm{H} 10 \mathrm{O} 2 \mathrm{~N}$ \\
\hline 164.1081 & 164.1081 & -0.13 & 4.5 & $\mathrm{C} 10 \mathrm{H} 14 \mathrm{ON}$ \\
\hline
\end{tabular}




\begin{tabular}{|c|c|c|c|c|}
\hline 165.0670 & 165.0670 & 0.15 & 5.5 & $\mathrm{C} 8 \mathrm{H} 9 \mathrm{O} 2 \mathrm{~N} 2$ \\
\hline 165.1034 & 165.1033 & 0.11 & 4.5 & C9 H13 O N2 \\
\hline 166.0510 & 166.0510 & 0.01 & 5.5 & $\mathrm{C} 8 \mathrm{H} 8 \mathrm{O} 3 \mathrm{~N}$ \\
\hline 166.0873 & 166.0874 & -0.44 & 4.5 & $\mathrm{C} 9 \mathrm{H} 12 \mathrm{O} 2 \mathrm{~N}$ \\
\hline 167.0826 & 167.0826 & -0.17 & 4.5 & $\mathrm{C} 8 \mathrm{H} 11 \mathrm{O} 2 \mathrm{~N} 2$ \\
\hline 168.0302 & 168.0302 & 0.04 & 5.5 & C7 H6 O4 N \\
\hline 168.0454 & 168.0455 & -0.26 & 9.5 & C11 H6 O N \\
\hline 168.0666 & 168.0666 & -0.17 & 4.5 & $\mathrm{C} 8 \mathrm{H} 10 \mathrm{O} 3 \mathrm{~N}$ \\
\hline 168.1030 & 168.1030 & -0.24 & 3.5 & $\mathrm{C} 9 \mathrm{H} 14 \mathrm{O} 2 \mathrm{~N}$ \\
\hline 169.0982 & 169.0983 & -0.20 & 3.5 & $\mathrm{C} 8 \mathrm{H} 13 \mathrm{O} 2 \mathrm{~N} 2$ \\
\hline 170.0458 & 170.0459 & -0.38 & 4.5 & $\mathrm{C} 7 \mathrm{H} 8 \mathrm{O} 4 \mathrm{~N}$ \\
\hline 170.0611 & 170.0611 & -0.16 & 8.5 & C11 H8 O N \\
\hline 170.0823 & 170.0823 & 0.27 & 3.5 & $\mathrm{C} 8 \mathrm{H} 12 \mathrm{O} 3 \mathrm{~N}$ \\
\hline 171.0565 & 171.0565 & 0.61 & 8.5 & C10 H7 O N2 \\
\hline 172.0404 & 172.0404 & 0.15 & 8.5 & $\mathrm{C} 10 \mathrm{H} 6 \mathrm{O} 2 \mathrm{~N}$ \\
\hline 172.0615 & 172.0615 & 0.06 & 3.5 & $\mathrm{C} 7 \mathrm{H} 10 \mathrm{O} 4 \mathrm{~N}$ \\
\hline 172.0768 & 172.0768 & -0.07 & 7.5 & C11 H10 O N \\
\hline 172.0979 & 172.0979 & 0.14 & 2.5 & $\mathrm{C} 8 \mathrm{H} 14 \mathrm{O} 3 \mathrm{~N}$ \\
\hline 173.0720 & 173.0720 & 0.05 & 7.5 & $\mathrm{C} 10 \mathrm{H} 9 \mathrm{O}$ N2 \\
\hline 174.0408 & 174.0408 & -0.02 & 3.5 & C6 H8 O5 N \\
\hline 174.0561 & 174.0561 & 0.11 & 7.5 & $\mathrm{C} 10 \mathrm{H} 8 \mathrm{O} 2 \mathrm{~N}$ \\
\hline 174.0772 & 174.0772 & -0.02 & 2.5 & $\mathrm{C} 7 \mathrm{H} 12 \mathrm{O} 4 \mathrm{~N}$ \\
\hline 174.0924 & 174.0924 & -0.16 & 6.5 & $\mathrm{C} 11 \mathrm{H} 12 \mathrm{ON}$ \\
\hline 175.0513 & 175.0513 & -0.10 & 7.5 & $\mathrm{C} 9 \mathrm{H} 7 \mathrm{O} 2 \mathrm{~N} 2$ \\
\hline 175.0877 & 175.0877 & -0.02 & 6.5 & C10 H11 O N2 \\
\hline 176.0353 & 176.0353 & 0.12 & 7.5 & C9 H6 O3 N \\
\hline 176.0717 & 176.0717 & 0.07 & 6.5 & $\mathrm{C} 10 \mathrm{H} 10 \mathrm{O} 2 \mathrm{~N}$ \\
\hline 176.1080 & 176.1081 & -0.39 & 5.5 & $\mathrm{C} 11 \mathrm{H} 14 \mathrm{ON}$ \\
\hline 177.0670 & 177.0670 & 0.21 & 6.5 & C9 H9 O2 N2 \\
\hline 178.0510 & 178.0510 & 0.21 & 6.5 & $\mathrm{C} 9 \mathrm{H} 8 \mathrm{O} 3 \mathrm{~N}$ \\
\hline 178.0874 & 178.0874 & 0.15 & 5.5 & $\mathrm{C} 10 \mathrm{H} 12 \mathrm{O} 2 \mathrm{~N}$ \\
\hline 178.1238 & 178.1237 & 0.47 & 4.5 & C11 H16 O N \\
\hline 179.0462 & 179.0462 & -0.34 & 6.5 & $\mathrm{C} 8 \mathrm{H} 7 \mathrm{O} 3 \mathrm{~N} 2$ \\
\hline 179.0827 & 179.0826 & 0.27 & 5.5 & $\mathrm{C} 9 \mathrm{H} 11 \mathrm{O} 2 \mathrm{~N} 2$ \\
\hline 179.1190 & 179.1190 & -0.12 & 4.5 & C10 H15 O N2 \\
\hline 180.0303 & 180.0302 & 0.37 & 6.5 & C8 H6 O4 N \\
\hline 180.0667 & 180.0666 & 0.27 & 5.5 & $\mathrm{C} 9 \mathrm{H} 10 \mathrm{O} 3 \mathrm{~N}$ \\
\hline 180.1030 & 180.1030 & 0.03 & 4.5 & $\mathrm{C} 10 \mathrm{H} 14 \mathrm{O} 2 \mathrm{~N}$ \\
\hline 181.0619 & 181.0619 & 0.27 & 5.5 & C8 H9 O3 N2 \\
\hline 181.0983 & 181.0983 & -0.02 & 4.5 & $\mathrm{C} 9 \mathrm{H} 13 \mathrm{O} 2 \mathrm{~N} 2$ \\
\hline 182.0459 & 182.0459 & 0.15 & 5.5 & $\mathrm{C} 8 \mathrm{H} 8 \mathrm{O} 4 \mathrm{~N}$ \\
\hline 182.0612 & 182.0611 & 0.08 & 9.5 & $\mathrm{C} 12 \mathrm{H} 8 \mathrm{O} \mathrm{N}$ \\
\hline 182.0822 & 182.0823 & -0.22 & 4.5 & $\mathrm{C} 9 \mathrm{H} 12 \mathrm{O} 3 \mathrm{~N}$ \\
\hline
\end{tabular}




\begin{tabular}{|c|c|c|c|c|}
\hline 182.1188 & 182.1187 & 0.92 & 3.5 & $\mathrm{C} 10 \mathrm{H} 16 \mathrm{O} 2 \mathrm{~N}$ \\
\hline 183.0776 & 183.0775 & 0.43 & 4.5 & C8 H11 O3 N2 \\
\hline 183.1140 & 183.1139 & 0.33 & 3.5 & $\mathrm{C} 9 \mathrm{H} 15 \mathrm{O} 2 \mathrm{~N} 2$ \\
\hline 184.0405 & 184.0405 & 0.32 & 9.5 & $\mathrm{C} 11 \mathrm{H} 6 \mathrm{O} 2 \mathrm{~N}$ \\
\hline 184.0615 & 184.0615 & -0.06 & 4.5 & $\mathrm{C} 8 \mathrm{H} 10 \mathrm{O} 4 \mathrm{~N}$ \\
\hline 184.0768 & 184.0769 & 0.18 & 8.5 & $\mathrm{C} 12 \mathrm{H} 10 \mathrm{ON}$ \\
\hline 184.0980 & 184.0979 & 0.26 & 3.5 & C9 H14 O3 N \\
\hline 185.0356 & 185.0357 & -0.45 & 9.5 & $\mathrm{C} 10 \mathrm{H} 5 \mathrm{O} 2 \mathrm{~N} 2$ \\
\hline 185.0720 & 185.0720 & 0.01 & 8.5 & C11 H9 O N2 \\
\hline 186.0561 & 186.0561 & 0.31 & 8.5 & $\mathrm{C} 11 \mathrm{H} 8 \mathrm{O} 2 \mathrm{~N}$ \\
\hline 186.0772 & 186.0772 & 0.10 & 3.5 & $\mathrm{C} 8 \mathrm{H} 12 \mathrm{O} 4 \mathrm{~N}$ \\
\hline 186.0925 & 186.0924 & 0.06 & 7.5 & $\mathrm{C} 12 \mathrm{H} 12 \mathrm{ON}$ \\
\hline 187.0512 & 187.0513 & -0.83 & 8.5 & $\mathrm{C} 10 \mathrm{H} 7 \mathrm{O} 2 \mathrm{~N} 2$ \\
\hline 187.0878 & 187.0877 & 0.38 & 7.5 & C11 H11 O N2 \\
\hline 188.0354 & 188.0354 & 0.39 & 8.5 & C10 H6 O3 N \\
\hline 188.0565 & 188.0565 & 0.51 & 3.5 & $\mathrm{C} 7 \mathrm{H} 10 \mathrm{O} 5 \mathrm{~N}$ \\
\hline 188.0718 & 188.0717 & 0.51 & 7.5 & $\mathrm{C} 11 \mathrm{H} 10 \mathrm{O} 2 \mathrm{~N}$ \\
\hline 188.0928 & 188.0928 & -0.14 & 2.5 & $\mathrm{C} 8 \mathrm{H} 14 \mathrm{O} 4 \mathrm{~N}$ \\
\hline 188.1082 & 188.1081 & 0.43 & 6.5 & $\mathrm{C} 12 \mathrm{H} 14 \mathrm{ON}$ \\
\hline 189.0670 & 189.0670 & 0.02 & 7.5 & $\mathrm{C} 10 \mathrm{H} 9 \mathrm{O} 2 \mathrm{~N} 2$ \\
\hline 189.1033 & 189.1033 & 0.04 & 6.5 & C11 H13 O N2 \\
\hline 190.0511 & 190.0510 & 0.64 & 7.5 & $\mathrm{C} 10 \mathrm{H} 8 \mathrm{O} 3 \mathrm{~N}$ \\
\hline 190.0874 & 190.0874 & 0.48 & 6.5 & $\mathrm{C} 11 \mathrm{H} 12 \mathrm{O} 2 \mathrm{~N}$ \\
\hline 191.0462 & 191.0462 & -0.07 & 7.5 & C9 H7 O3 N2 \\
\hline 191.0826 & 191.0826 & 0.09 & 6.5 & $\mathrm{C} 10 \mathrm{H} 11 \mathrm{O} 2 \mathrm{~N} 2$ \\
\hline 192.0303 & 192.0302 & 0.33 & 7.5 & C9 H6 O4 N \\
\hline 192.0667 & 192.0666 & 0.56 & 6.5 & $\mathrm{C} 10 \mathrm{H} 10 \mathrm{O} 3 \mathrm{~N}$ \\
\hline 192.1030 & 192.1030 & 0.09 & 5.5 & $\mathrm{C} 11 \mathrm{H} 14 \mathrm{O} 2 \mathrm{~N}$ \\
\hline 193.0621 & 193.0619 & 1.25 & 6.5 & C9 H9 O3 N2 \\
\hline 193.0983 & 193.0983 & 0.15 & 5.5 & $\mathrm{C} 10 \mathrm{H} 13 \mathrm{O} 2 \mathrm{~N} 2$ \\
\hline 193.1347 & 193.1346 & 0.28 & 4.5 & C11 H17 O N2 \\
\hline 194.0459 & 194.0459 & 0.20 & 6.5 & C9 H8 O4 N \\
\hline 194.0612 & 194.0612 & 0.22 & 10.5 & $\mathrm{C} 13 \mathrm{H} 8 \mathrm{ON}$ \\
\hline 194.0824 & 194.0823 & 0.64 & 5.5 & $\mathrm{C} 10 \mathrm{H} 12 \mathrm{O} 3 \mathrm{~N}$ \\
\hline 195.0774 & 195.0775 & -0.39 & 5.5 & C9 H11 O3 N2 \\
\hline 195.1139 & 195.1139 & -0.02 & 4.5 & $\mathrm{C} 10 \mathrm{H} 15 \mathrm{O} 2 \mathrm{~N} 2$ \\
\hline 196.0614 & 196.0615 & -0.50 & 5.5 & C9 H10 O4 N \\
\hline 196.0768 & 196.0768 & 0.13 & 9.5 & $\mathrm{C} 13 \mathrm{H} 10 \mathrm{ON}$ \\
\hline 196.0979 & 196.0979 & -0.20 & 4.5 & $\mathrm{C} 10 \mathrm{H} 14 \mathrm{O} 3 \mathrm{~N}$ \\
\hline 197.0724 & 197.0721 & 2.00 & 9.5 & C12 H9 O N2 \\
\hline 197.0930 & 197.0932 & -0.81 & 4.5 & $\mathrm{C} 9 \mathrm{H} 13 \mathrm{O} 3 \mathrm{~N} 2$ \\
\hline 197.1296 & 197.1296 & 0.26 & 3.5 & $\mathrm{C} 10 \mathrm{H} 17 \mathrm{O} 2 \mathrm{~N} 2$ \\
\hline 198.0408 & 198.0408 & 0.09 & 5.5 & C8 H8 O5 N \\
\hline
\end{tabular}




\begin{tabular}{|c|c|c|c|c|}
\hline 198.0561 & 198.0561 & 0.40 & 9.5 & $\mathrm{C} 12 \mathrm{H} 8 \mathrm{O} 2 \mathrm{~N}$ \\
\hline 198.0773 & 198.0772 & 0.44 & 4.5 & C9 H12 O4 N \\
\hline 198.0925 & 198.0925 & 0.42 & 8.5 & $\mathrm{C} 13 \mathrm{H} 12 \mathrm{ON}$ \\
\hline 198.1136 & 198.1136 & 0.24 & 3.5 & $\mathrm{C} 10 \mathrm{H} 16 \mathrm{O} 3 \mathrm{~N}$ \\
\hline 199.0513 & 199.0513 & -0.22 & 9.5 & $\mathrm{C} 11 \mathrm{H} 7 \mathrm{O} 2 \mathrm{~N} 2$ \\
\hline 199.0725 & 199.0724 & 0.43 & 4.5 & C8 H11 O4 N2 \\
\hline 199.0878 & 199.0878 & 0.37 & 8.5 & C12 H11 O N2 \\
\hline 200.0566 & 200.0565 & 0.67 & 4.5 & C8 H10 O5 N \\
\hline 200.0717 & 200.0717 & -0.21 & 8.5 & $\mathrm{C} 12 \mathrm{H} 10 \mathrm{O} 2 \mathrm{~N}$ \\
\hline 200.0929 & 200.0928 & 0.45 & 3.5 & C9 H14 O4 N \\
\hline 200.1082 & 200.1081 & 0.50 & 7.5 & $\mathrm{C} 13 \mathrm{H} 14 \mathrm{ON}$ \\
\hline 200.1293 & 200.1292 & 0.63 & 2.5 & $\mathrm{C} 10 \mathrm{H} 18 \mathrm{O} 3 \mathrm{~N}$ \\
\hline 201.0670 & 201.0670 & 0.04 & 8.5 & $\mathrm{C} 11 \mathrm{H} 9 \mathrm{O} 2 \mathrm{~N} 2$ \\
\hline 201.1033 & 201.1033 & -0.15 & 7.5 & C12 H13 O N2 \\
\hline 202.0509 & 202.0510 & -0.13 & 8.5 & $\mathrm{C} 11 \mathrm{H} 8 \mathrm{O} 3 \mathrm{~N}$ \\
\hline 202.0721 & 202.0721 & -0.15 & 3.5 & $\mathrm{C} 8 \mathrm{H} 12 \mathrm{O} 5 \mathrm{~N}$ \\
\hline 202.0874 & 202.0874 & 0.03 & 7.5 & $\mathrm{C} 12 \mathrm{H} 12 \mathrm{O} 2 \mathrm{~N}$ \\
\hline 202.1086 & 202.1085 & 0.38 & 2.5 & C9 H16 O4 N \\
\hline 203.0462 & 203.0462 & -0.31 & 8.5 & $\mathrm{C} 10 \mathrm{H} 7 \mathrm{O} 3 \mathrm{~N} 2$ \\
\hline 203.0827 & 203.0826 & 0.28 & 7.5 & $\mathrm{C} 11 \mathrm{H} 11 \mathrm{O} 2 \mathrm{~N} 2$ \\
\hline 204.0302 & 204.0302 & -0.07 & 8.5 & C10 H6 O4 N \\
\hline 204.0667 & 204.0666 & 0.48 & 7.5 & C11 H10 O3 N \\
\hline 204.1029 & 204.1030 & -0.34 & 6.5 & $\mathrm{C} 12 \mathrm{H} 14 \mathrm{O} 2 \mathrm{~N}$ \\
\hline 205.0619 & 205.0619 & 0.39 & 7.5 & $\mathrm{C} 10 \mathrm{H} 9 \mathrm{O} 3 \mathrm{~N} 2$ \\
\hline 205.0982 & 205.0983 & -0.17 & 6.5 & $\mathrm{C} 11 \mathrm{H} 13 \mathrm{O} 2 \mathrm{~N} 2$ \\
\hline 206.0459 & 206.0459 & 0.08 & 7.5 & $\mathrm{C} 10 \mathrm{H} 8 \mathrm{O} 4 \mathrm{~N}$ \\
\hline 206.0823 & 206.0823 & 0.07 & 6.5 & $\mathrm{C} 11 \mathrm{H} 12 \mathrm{O} 3 \mathrm{~N}$ \\
\hline 206.1188 & 206.1187 & 0.50 & 5.5 & $\mathrm{C} 12 \mathrm{H} 16 \mathrm{O} 2 \mathrm{~N}$ \\
\hline 207.0043 & 207.0047 & -2.25 & 8.5 & C8 H3 O5 N2 \\
\hline 207.0775 & 207.0775 & 0.09 & 6.5 & $\mathrm{C} 10 \mathrm{H} 11 \mathrm{O} 3 \mathrm{~N} 2$ \\
\hline 207.1139 & 207.1139 & 0.17 & 5.5 & $\mathrm{C} 11 \mathrm{H} 15 \mathrm{O} 2 \mathrm{~N} 2$ \\
\hline 208.0616 & 208.0615 & 0.13 & 6.5 & $\mathrm{C} 10 \mathrm{H} 10 \mathrm{O} 4 \mathrm{~N}$ \\
\hline 208.0770 & 208.0769 & 0.88 & 10.5 & $\mathrm{C} 14 \mathrm{H} 10 \mathrm{ON}$ \\
\hline 208.0978 & 208.0979 & -0.44 & 5.5 & $\mathrm{C} 11 \mathrm{H} 14 \mathrm{O} 3 \mathrm{~N}$ \\
\hline 209.0931 & 209.0932 & -0.49 & 5.5 & $\mathrm{C} 10 \mathrm{H} 13 \mathrm{O} 3 \mathrm{~N} 2$ \\
\hline 209.1296 & 209.1296 & 0.15 & 4.5 & $\mathrm{C} 11 \mathrm{H} 17 \mathrm{O} 2 \mathrm{~N} 2$ \\
\hline 210.0409 & 210.0408 & 0.64 & 6.5 & C9 H8 O5 N \\
\hline 210.0560 & 210.0561 & -0.20 & 10.5 & $\mathrm{C} 13 \mathrm{H} 8 \mathrm{O} 2 \mathrm{~N}$ \\
\hline 210.0773 & 210.0772 & 0.73 & 5.5 & $\mathrm{C} 10 \mathrm{H} 12 \mathrm{O} 4 \mathrm{~N}$ \\
\hline 210.0926 & 210.0925 & 0.63 & 9.5 & $\mathrm{C} 14 \mathrm{H} 12 \mathrm{O} \mathrm{N}$ \\
\hline 210.1137 & 210.1136 & 0.81 & 4.5 & C11 H16 O3 N \\
\hline 211.0726 & 211.0724 & 1.01 & 5.5 & C9 H11 O4 N2 \\
\hline 211.0880 & 211.0878 & 1.39 & 9.5 & C13 H11 O N2 \\
\hline
\end{tabular}




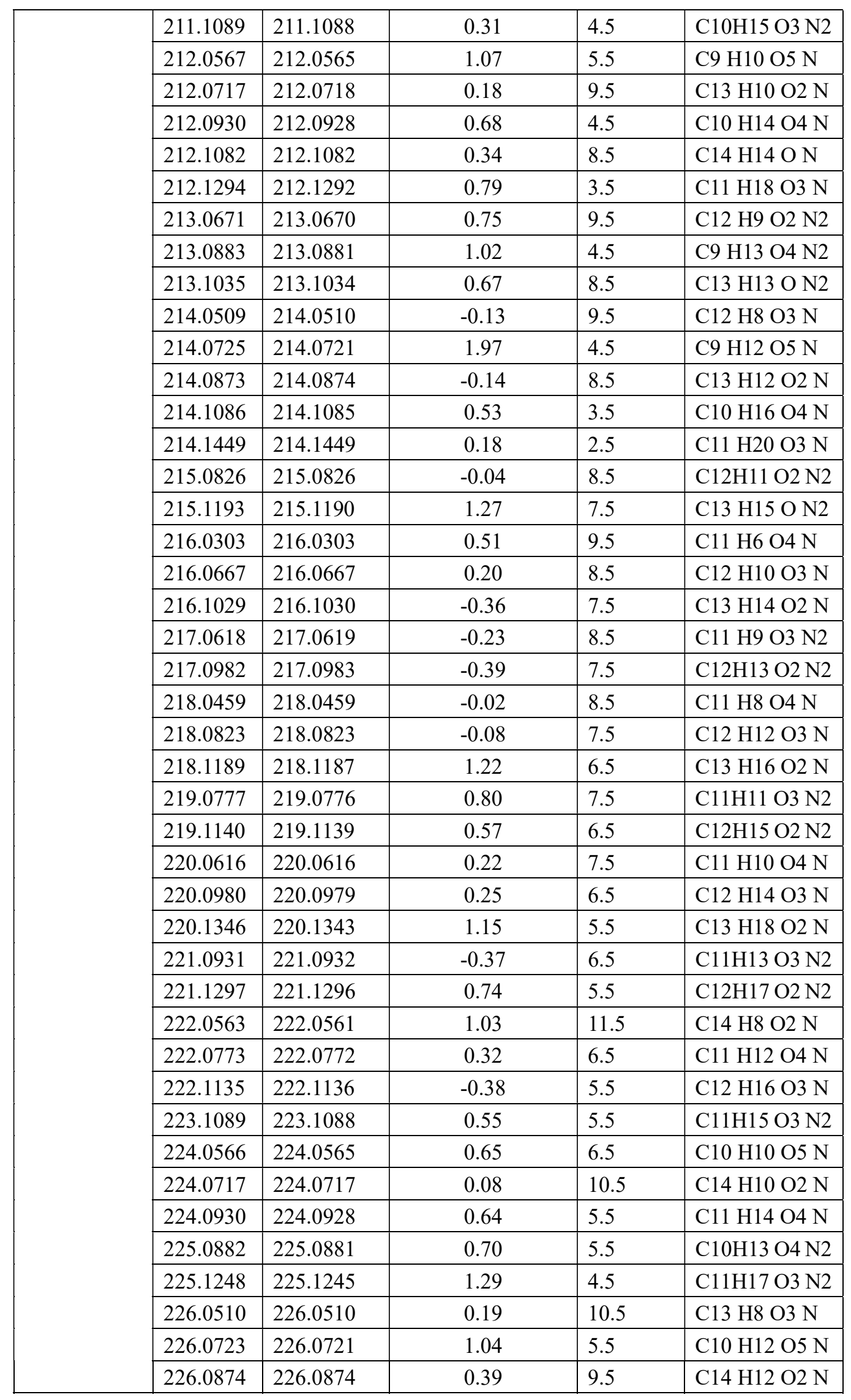




\begin{tabular}{|c|c|c|c|c|}
\hline 226.1084 & 226.1085 & -0.46 & 4.5 & C11 H16 O4 N \\
\hline 226.1200 & 226.1197 & 1.05 & 4.5 & $\mathrm{C} 10 \mathrm{H} 16 \mathrm{O} 3 \mathrm{~N} 3$ \\
\hline 226.1451 & 226.1449 & 0.90 & 3.5 & $\mathrm{C} 12 \mathrm{H} 20 \mathrm{O} 3 \mathrm{~N}$ \\
\hline 227.0827 & 227.0827 & 0.48 & 9.5 & $\mathrm{C} 13 \mathrm{H} 11 \mathrm{O} 2 \mathrm{~N} 2$ \\
\hline 227.1038 & 227.1037 & 0.35 & 4.5 & $\mathrm{C} 10 \mathrm{H} 15 \mathrm{O} 4 \mathrm{~N} 2$ \\
\hline 228.0153 & 228.0153 & 1.38 & 6.5 & C8 H6 O7 N \\
\hline 228.0668 & 228.0667 & 0.59 & 9.5 & $\mathrm{C} 13 \mathrm{H} 10 \mathrm{O} 3 \mathrm{~N}$ \\
\hline 228.0882 & 228.0878 & 1.89 & 4.5 & $\mathrm{C} 10 \mathrm{H} 14 \mathrm{O} 5 \mathrm{~N}$ \\
\hline 228.1031 & 228.1031 & 0.56 & 8.5 & $\mathrm{C} 14 \mathrm{H} 14 \mathrm{O} 2 \mathrm{~N}$ \\
\hline 228.1244 & 228.1241 & 1.12 & 3.5 & C11 H18 O4 N \\
\hline 229.0985 & 229.0983 & 0.96 & 8.5 & $\mathrm{C} 13 \mathrm{H} 13 \mathrm{O} 2 \mathrm{~N} 2$ \\
\hline 230.0462 & 230.0459 & 1.30 & 9.5 & $\mathrm{C} 12 \mathrm{H} 8 \mathrm{O} 4 \mathrm{~N}$ \\
\hline 230.0823 & 230.0823 & 0.32 & 8.5 & $\mathrm{C} 13 \mathrm{H} 12 \mathrm{O} 3 \mathrm{~N}$ \\
\hline 230.1189 & 230.1187 & 1.08 & 7.5 & $\mathrm{C} 14 \mathrm{H} 16 \mathrm{O} 2 \mathrm{~N}$ \\
\hline 231.0777 & 231.0776 & 0.58 & 8.5 & $\mathrm{C} 12 \mathrm{H} 11 \mathrm{O} 3 \mathrm{~N} 2$ \\
\hline 231.1139 & 231.1139 & -0.22 & 7.5 & $\mathrm{C} 13 \mathrm{H} 15 \mathrm{O} 2 \mathrm{~N} 2$ \\
\hline 232.0617 & 232.0616 & 0.56 & 8.5 & $\mathrm{C} 12 \mathrm{H} 10 \mathrm{O} 4 \mathrm{~N}$ \\
\hline 232.0981 & 232.0979 & 0.60 & 7.5 & $\mathrm{C} 13 \mathrm{H} 14 \mathrm{O} 3 \mathrm{~N}$ \\
\hline 232.1345 & 232.1343 & 0.96 & 6.5 & $\mathrm{C} 14 \mathrm{H} 18 \mathrm{O} 2 \mathrm{~N}$ \\
\hline 233.0934 & 233.0932 & 1.09 & 7.5 & $\mathrm{C} 12 \mathrm{H} 13 \mathrm{O} 3 \mathrm{~N} 2$ \\
\hline 234.0411 & 234.0409 & 1.39 & 8.5 & $\mathrm{C} 11 \mathrm{H} 8 \mathrm{O} 5 \mathrm{~N}$ \\
\hline 234.0773 & 234.0772 & 0.47 & 7.5 & $\mathrm{C} 12 \mathrm{H} 12 \mathrm{O} 4 \mathrm{~N}$ \\
\hline 234.1136 & 234.1136 & 0.09 & 6.5 & $\mathrm{C} 13 \mathrm{H} 16 \mathrm{O} 3 \mathrm{~N}$ \\
\hline 235.0723 & 235.0724 & -0.49 & 7.5 & $\mathrm{C} 11 \mathrm{H} 11 \mathrm{O} 4 \mathrm{~N} 2$ \\
\hline 235.1089 & 235.1088 & 0.34 & 6.5 & $\mathrm{C} 12 \mathrm{H} 15 \mathrm{O} 3 \mathrm{~N} 2$ \\
\hline 236.0565 & 236.0565 & 0.31 & 7.5 & $\mathrm{C} 11 \mathrm{H} 10 \mathrm{O} 5 \mathrm{~N}$ \\
\hline 236.0928 & 236.0928 & -0.02 & 6.5 & $\mathrm{C} 12 \mathrm{H} 14 \mathrm{O} 4 \mathrm{~N}$ \\
\hline 236.1295 & 236.1292 & 1.27 & 5.5 & $\mathrm{C} 13 \mathrm{H} 18 \mathrm{O} 3 \mathrm{~N}$ \\
\hline 237.0882 & 237.0881 & 0.55 & 6.5 & $\mathrm{C} 11 \mathrm{H} 13 \mathrm{O} 4 \mathrm{~N} 2$ \\
\hline 237.1248 & 237.1245 & 1.32 & 5.5 & $\mathrm{C} 12 \mathrm{H} 17 \mathrm{O} 3 \mathrm{~N} 2$ \\
\hline 238.0723 & 238.0721 & 1.00 & 6.5 & C11 H12 O5 N \\
\hline 238.1087 & 238.1085 & 1.03 & 5.5 & $\mathrm{C} 12 \mathrm{H} 16 \mathrm{O} 4 \mathrm{~N}$ \\
\hline 239.1039 & 239.1037 & 0.90 & 5.5 & $\mathrm{C} 11 \mathrm{H} 15 \mathrm{O} 4 \mathrm{~N} 2$ \\
\hline 240.0669 & 240.0667 & 1.26 & 10.5 & $\mathrm{C} 14 \mathrm{H} 10 \mathrm{O} 3 \mathrm{~N}$ \\
\hline 240.0882 & 240.0878 & 1.73 & 5.5 & C11 H14 O5 N \\
\hline 240.1032 & 240.1031 & 0.74 & 9.5 & $\mathrm{C} 15 \mathrm{H} 14 \mathrm{O} 2 \mathrm{~N}$ \\
\hline 241.0986 & 241.0983 & 1.41 & 9.5 & $\mathrm{C} 14 \mathrm{H} 13 \mathrm{O} 2 \mathrm{~N} 2$ \\
\hline 241.1195 & 241.1194 & 0.42 & 4.5 & $\mathrm{C} 11 \mathrm{H} 17 \mathrm{O} 4 \mathrm{~N} 2$ \\
\hline 242.0675 & 242.0674 & 1.86 & 5.5 & $\mathrm{C} 10 \mathrm{H} 12 \mathrm{O} 6 \mathrm{~N}$ \\
\hline 242.0824 & 242.0823 & 0.59 & 9.5 & $\mathrm{C} 14 \mathrm{H} 12 \mathrm{O} 3 \mathrm{~N}$ \\
\hline 242.1192 & 242.1187 & 2.10 & 8.5 & $\mathrm{C} 15 \mathrm{H} 16 \mathrm{O} 2 \mathrm{~N}$ \\
\hline 242.1763 & 242.1762 & 0.44 & 2.5 & $\mathrm{C} 13 \mathrm{H} 24 \mathrm{O} 3 \mathrm{~N}$ \\
\hline 243.0778 & 243.0776 & 1.17 & 9.5 & $\mathrm{C} 13 \mathrm{H} 11 \mathrm{O} 3 \mathrm{~N} 2$ \\
\hline
\end{tabular}




\begin{tabular}{|c|c|c|c|c|}
\hline 243.1142 & 243.1140 & 1.07 & 8.5 & $\mathrm{C} 14 \mathrm{H} 15 \mathrm{O} 2 \mathrm{~N} 2$ \\
\hline 244.0618 & 244.0616 & 1.14 & 9.5 & C13 H10 O4 N \\
\hline 244.0981 & 244.0980 & 0.67 & 8.5 & $\mathrm{C} 14 \mathrm{H} 14 \mathrm{O} 3 \mathrm{~N}$ \\
\hline 245.0934 & 245.0932 & 1.04 & 8.5 & $\mathrm{C} 13 \mathrm{H} 13 \mathrm{O} 3 \mathrm{~N} 2$ \\
\hline 246.0773 & 246.0772 & 0.61 & 8.5 & $\mathrm{C} 13 \mathrm{H} 12 \mathrm{O} 4 \mathrm{~N}$ \\
\hline 246.1138 & 246.1136 & 0.91 & 7.5 & $\mathrm{C} 14 \mathrm{H} 16 \mathrm{O} 3 \mathrm{~N}$ \\
\hline 247.1091 & 247.1088 & 1.31 & 7.5 & $\mathrm{C} 13 \mathrm{H} 15 \mathrm{O} 3 \mathrm{~N} 2$ \\
\hline 248.0568 & 248.0565 & 1.39 & 8.5 & $\mathrm{C} 12 \mathrm{H} 10 \mathrm{O} 5 \mathrm{~N}$ \\
\hline 248.0931 & 248.0928 & 0.91 & 7.5 & $\mathrm{C} 13 \mathrm{H} 14 \mathrm{O} 4 \mathrm{~N}$ \\
\hline 248.1296 & 248.1292 & 1.52 & 6.5 & $\mathrm{C} 14 \mathrm{H} 18 \mathrm{O} 3 \mathrm{~N}$ \\
\hline 249.0883 & 249.0881 & 0.75 & 7.5 & $\mathrm{C} 12 \mathrm{H} 13 \mathrm{O} 4 \mathrm{~N} 2$ \\
\hline 249.1247 & 249.1245 & 1.06 & 6.5 & $\mathrm{C} 13 \mathrm{H} 17 \mathrm{O} 3 \mathrm{~N} 2$ \\
\hline 250.0727 & 250.0730 & 2.34 & 7.5 & $\mathrm{C} 12 \mathrm{H} 12 \mathrm{O} 5 \mathrm{~N}$ \\
\hline 250.1087 & 250.1085 & 0.99 & 6.5 & $\mathrm{C} 13 \mathrm{H} 16 \mathrm{O} 4 \mathrm{~N}$ \\
\hline 250.1452 & 250.1449 & 1.23 & 5.5 & $\mathrm{C} 14 \mathrm{H} 20 \mathrm{O} 3 \mathrm{~N}$ \\
\hline 251.1039 & 251.1037 & 0.68 & 6.5 & $\mathrm{C} 12 \mathrm{H} 15 \mathrm{O} 4 \mathrm{~N} 2$ \\
\hline 252.0879 & 252.0878 & 0.68 & 6.5 & $\mathrm{C} 12 \mathrm{H} 14 \mathrm{O} 5 \mathrm{~N}$ \\
\hline 254.0668 & 254.0670 & -0.66 & 6.5 & $\mathrm{C} 11 \mathrm{H} 12 \mathrm{O} 6 \mathrm{~N}$ \\
\hline 254.0825 & 254.0823 & 0.80 & 10.5 & $\mathrm{C} 15 \mathrm{H} 12 \mathrm{O} 3 \mathrm{~N}$ \\
\hline 254.1038 & 254.1034 & 1.38 & 5.5 & $\mathrm{C} 12 \mathrm{H} 16 \mathrm{O} 5 \mathrm{~N}$ \\
\hline 255.1142 & 255.1140 & 1.21 & 9.5 & $\mathrm{C} 15 \mathrm{H} 15 \mathrm{O} 2 \mathrm{~N} 2$ \\
\hline 255.1355 & 255.1350 & 1.79 & 4.5 & $\mathrm{C} 12 \mathrm{H} 19 \mathrm{O} 4 \mathrm{~N} 2$ \\
\hline 256.0620 & 256.0625 & 1.99 & 10.5 & $\mathrm{C} 14 \mathrm{H} 10 \mathrm{O} 4 \mathrm{~N}$ \\
\hline 256.0982 & 256.0980 & 1.11 & 9.5 & C15 H14 O3 N \\
\hline 257.0934 & 257.0932 & 0.83 & 9.5 & $\mathrm{C} 14 \mathrm{H} 13 \mathrm{O} 3 \mathrm{~N} 2$ \\
\hline 257.1300 & 257.1296 & 1.82 & 8.5 & $\mathrm{C} 15 \mathrm{H} 17 \mathrm{O} 2 \mathrm{~N} 2$ \\
\hline 258.0775 & 258.0772 & 1.24 & 9.5 & $\mathrm{C} 14 \mathrm{H} 12 \mathrm{O} 4 \mathrm{~N}$ \\
\hline 258.1140 & 258.1136 & 1.60 & 8.5 & $\mathrm{C} 15 \mathrm{H} 16 \mathrm{O} 3 \mathrm{~N}$ \\
\hline 259.1090 & 259.1089 & 0.75 & 8.5 & $\mathrm{C} 14 \mathrm{H} 15 \mathrm{O} 3 \mathrm{~N} 2$ \\
\hline 260.0931 & 260.0929 & 0.84 & 8.5 & $\mathrm{C} 14 \mathrm{H} 14 \mathrm{O} 4 \mathrm{~N}$ \\
\hline 260.1296 & 260.1292 & 1.29 & 7.5 & C15 H18 O3 N \\
\hline 261.0886 & 261.0881 & 2.11 & 8.5 & $\mathrm{C} 13 \mathrm{H} 13 \mathrm{O} 4 \mathrm{~N} 2$ \\
\hline 262.0724 & 262.0722 & 1.16 & 8.5 & $\mathrm{C} 13 \mathrm{H} 12 \mathrm{O} 5 \mathrm{~N}$ \\
\hline 262.1089 & 262.1085 & 1.59 & 7.5 & $\mathrm{C} 14 \mathrm{H} 16 \mathrm{O} 4 \mathrm{~N}$ \\
\hline 264.0879 & 264.0878 & 0.55 & 7.5 & $\mathrm{C} 13 \mathrm{H} 14 \mathrm{O} 5 \mathrm{~N}$ \\
\hline 264.1246 & 264.1241 & 1.71 & 6.5 & $\mathrm{C} 14 \mathrm{H} 18 \mathrm{O} 4 \mathrm{~N}$ \\
\hline 266.1040 & 266.1043 & 2.27 & 6.5 & $\mathrm{C} 13 \mathrm{H} 16 \mathrm{O} 5 \mathrm{~N}$ \\
\hline 266.1517 & 266.1513 & 2.50 & 5.5 & $\mathrm{C} 13 \mathrm{H} 20 \mathrm{O} 3 \mathrm{~N} 3$ \\
\hline 268.0985 & 268.0989 & 2.10 & 10.5 & C16 H14 O3 N \\
\hline 269.1512 & 269.1507 & 1.77 & 4.5 & $\mathrm{C} 13 \mathrm{H} 21 \mathrm{O} 4 \mathrm{~N} 2$ \\
\hline 270.1141 & 270.1145 & 2.01 & 9.5 & $\mathrm{C} 16 \mathrm{H} 16 \mathrm{O} 3 \mathrm{~N}$ \\
\hline 271.1091 & 271.1089 & 1.16 & 9.5 & $\mathrm{C} 15 \mathrm{H} 15 \mathrm{O} 3 \mathrm{~N} 2$ \\
\hline 272.0932 & 272.0929 & 1.54 & 9.5 & $\mathrm{C} 15 \mathrm{H} 14 \mathrm{O} 4 \mathrm{~N}$ \\
\hline
\end{tabular}




\begin{tabular}{|c|c|c|c|c|c|}
\hline & 273.1253 & 273.1261 & 3.09 & 8.5 & $\mathrm{C} 15 \mathrm{H} 17 \mathrm{O} 3 \mathrm{~N} 2$ \\
\hline & 274.0724 & 274.0722 & 1.04 & 9.5 & $\mathrm{C} 14 \mathrm{H} 12 \mathrm{O} 5 \mathrm{~N}$ \\
\hline & 274.1090 & 274.1085 & 1.82 & 8.5 & C15 H16 O4 N \\
\hline & 275.1046 & 275.1053 & 3.02 & 8.5 & $\mathrm{C} 14 \mathrm{H} 15 \mathrm{O} 4 \mathrm{~N} 2$ \\
\hline & 276.0882 & 276.0878 & 1.39 & 8.5 & C14 H14 O5 N \\
\hline & 276.1245 & 276.1241 & 1.16 & 7.5 & C15 H18 O4 N \\
\hline & 278.1038 & 278.1034 & 1.48 & 7.5 & $\mathrm{C} 14 \mathrm{H} 16 \mathrm{O} 5 \mathrm{~N}$ \\
\hline & 282.1141 & 282.1136 & 1.71 & 10.5 & $\mathrm{C} 17 \mathrm{H} 16 \mathrm{O} 3 \mathrm{~N}$ \\
\hline & 286.1090 & 286.1094 & 1.81 & 9.5 & C16 H16 O4 N \\
\hline & 288.0884 & 288.0886 & 2.20 & 9.5 & $\mathrm{C} 15 \mathrm{H} 14 \mathrm{O} 5 \mathrm{~N}$ \\
\hline & 290.1038 & 290.1035 & 1.36 & 8.5 & $\mathrm{C} 15 \mathrm{H} 16 \mathrm{O} 5 \mathrm{~N}$ \\
\hline & 300.1248 & 300.1251 & 2.16 & 9.5 & $\mathrm{C} 17 \mathrm{H} 18 \mathrm{O} 4 \mathrm{~N}$ \\
\hline & 302.1043 & 302.1043 & 2.93 & 9.5 & $\mathrm{C} 16 \mathrm{H} 16 \mathrm{O} 5 \mathrm{~N}$ \\
\hline \multirow{30}{*}{ Other } & 77.0395 & 77.0397 & -2.65 & 4.5 & C6 H5 \\
\hline & 91.0552 & 91.0553 & -1.75 & 4.5 & C7 H7 \\
\hline & 92.0504 & 92.0506 & -1.56 & 4.5 & C6 H6 N \\
\hline & 102.0348 & 102.0349 & -1.28 & 6.5 & C7 H4 N \\
\hline & 103.0551 & 103.0553 & -1.81 & 5.5 & $\mathrm{C} 8 \mathrm{H} 7$ \\
\hline & 105.0708 & 105.0710 & -1.49 & 4.5 & $\mathrm{C} 8 \mathrm{H} 9$ \\
\hline & 109.0770 & 109.0771 & -1.40 & 3.5 & C6 H9 N2 \\
\hline & 117.0709 & 117.0710 & -0.81 & 5.5 & C9 H9 \\
\hline & 119.0865 & 119.0866 & -1.06 & 4.5 & C9 H11 \\
\hline & 121.0770 & 121.0771 & -0.89 & 4.5 & C7 H9 N2 \\
\hline & 123.0926 & 123.0928 & -1.13 & 3.5 & C7 H11 N2 \\
\hline & 132.0486 & 132.0489 & -1.85 & 1.5 & C5 H10 O N S \\
\hline & 133.0770 & 133.0771 & -1.05 & 5.5 & C8 H9 N2 \\
\hline & 133.9913 & 133.9912 & -3.56 & 2.5 & $\mathrm{C} 3 \mathrm{H} 4 \mathrm{O} 3 \mathrm{~N} \mathrm{~S}$ \\
\hline & 137.9863 & 137.9861 & -2.62 & 1.5 & $\mathrm{C} 2 \mathrm{H} 4 \mathrm{O} 4 \mathrm{~N} \mathrm{~S}$ \\
\hline & 141.0457 & 141.0458 & -0.70 & 8.5 & C9 H5 N2 \\
\hline & 145.0770 & 145.0771 & -0.67 & 6.5 & C9 H9 N2 \\
\hline & 147.0927 & 147.0928 & -0.42 & 5.5 & C9 H11 N2 \\
\hline & 149.0427 & 149.0430 & -2.33 & 5.5 & C9 H9 S \\
\hline & 155.0614 & 155.0615 & -0.24 & 8.5 & $\mathrm{C} 10 \mathrm{H} 7 \mathrm{~N} 2$ \\
\hline & 159.0927 & 159.0928 & -0.37 & 6.5 & C10 H11 N2 \\
\hline & 160.0880 & 160.0880 & -0.29 & 6.5 & C9 H10 N3 \\
\hline & 164.0023 & 164.0023 & 0.02 & 2.5 & C4 H6 O4 N S \\
\hline & 166.0661 & 166.0662 & -1.05 & 9.5 & $\mathrm{C} 12 \mathrm{H} 8 \mathrm{~N}$ \\
\hline & 167.0615 & 167.0615 & -0.12 & 9.5 & C11 H7 N2 \\
\hline & 169.0117 & 169.0117 & -0.35 & 9.5 & C11 H5 S \\
\hline & 169.0772 & 169.0772 & 0.35 & 8.5 & C11 H9 N2 \\
\hline & 171.0274 & 171.0274 & -0.08 & 8.5 & $\mathrm{C} 11 \mathrm{H} 7 \mathrm{~S}$ \\
\hline & 173.1084 & 173.1084 & 0.04 & 6.5 & C11 H13 N2 \\
\hline & 173.9867 & 173.9867 & 0.27 & 4.5 & $\mathrm{C} 5 \mathrm{H} 4 \mathrm{O} 4 \mathrm{~N} \mathrm{~S}$ \\
\hline
\end{tabular}




\begin{tabular}{|l|l|l|l|l|l|}
\hline & 180.0334 & 180.0336 & -1.37 & 1.5 & C5H10 O4 N S \\
\cline { 2 - 6 } & 181.0772 & 181.0772 & 0.60 & 9.5 & C12 H9 N2 \\
\cline { 2 - 6 } & 183.0274 & 183.0274 & -0.05 & 9.5 & C12 H7 S \\
\cline { 2 - 6 } & 185.0430 & 185.0430 & -0.16 & 8.5 & C12 H9 S \\
\cline { 2 - 6 } & 187.0181 & 187.0183 & -0.91 & 4.5 & C6H7 O3 N2 S \\
\cline { 2 - 6 } & 230.0123 & 230.0124 & -2.38 & 5.5 & C8 H8 O5 N S \\
\cline { 2 - 6 } & 239.0332 & 239.0332 & -4.73 & 2.5 & C6H11O6N2 S \\
\cline { 2 - 6 } & 287.0931 & 287.0934 & -1.09 & 8.5 & C17 H19 S2 \\
\cline { 2 - 6 } & 289.9800 & 289.9800 & 0.65 & 6.5 & C9H8 O6 N S2 \\
\cline { 2 - 6 } & 361.9823 & 361.9824 & -0.21 & 8.5 & C11H8O11 N S \\
\hline
\end{tabular}




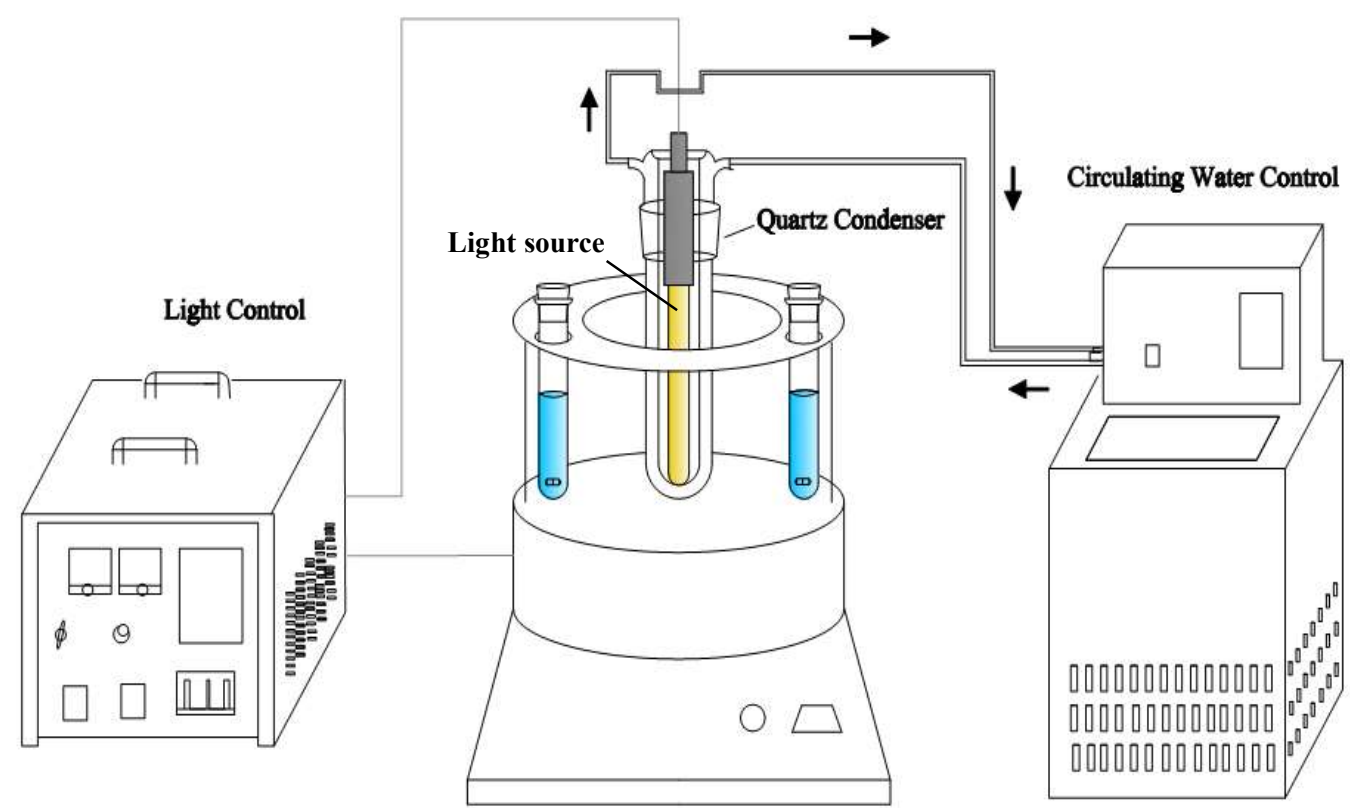

Figure S1: Experimental setup for the photolysis of the samples in the aqueous phase. Each extract was distributed into three tubes that corresponded to three different irradiation times, i.e. 0,4 , and $12 \mathrm{~h}$, with no oxidants added externally throughout the whole photolytic process. At each irradiation time point (e.g., 0 and $4 \mathrm{~h}$ ), the related tubes were wrapped with aluminum foil, and placed at the initial location until the end of 12-h photolysis. 

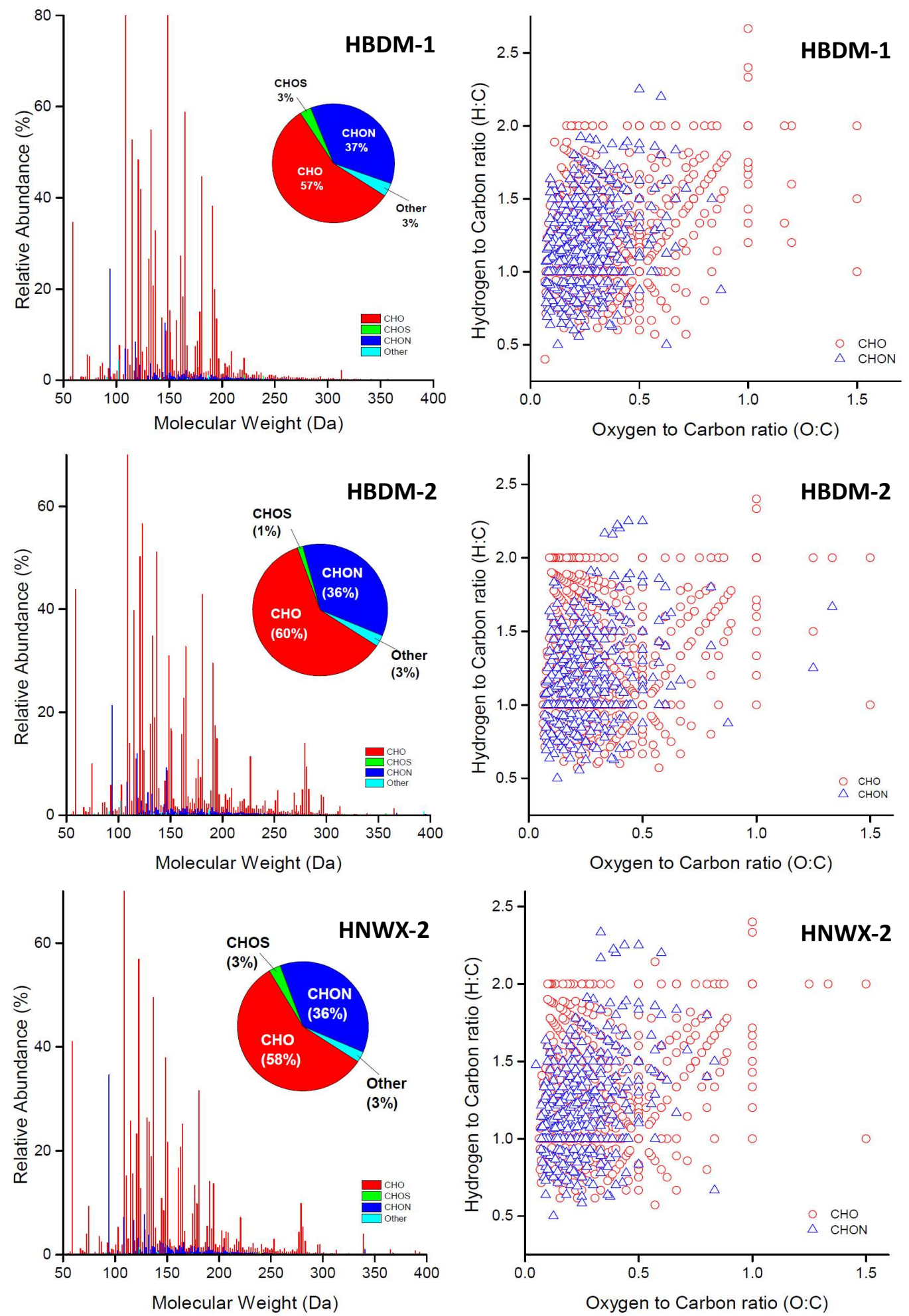

Figure S2: Reconstructed mass spectra for identified ions and Van Krevelen diagrams of $\mathrm{CHO}$ and $\mathrm{CHON}$ species in WSOC extracts from HBDM-1, HBDM-2 and HNWX2 samples. 


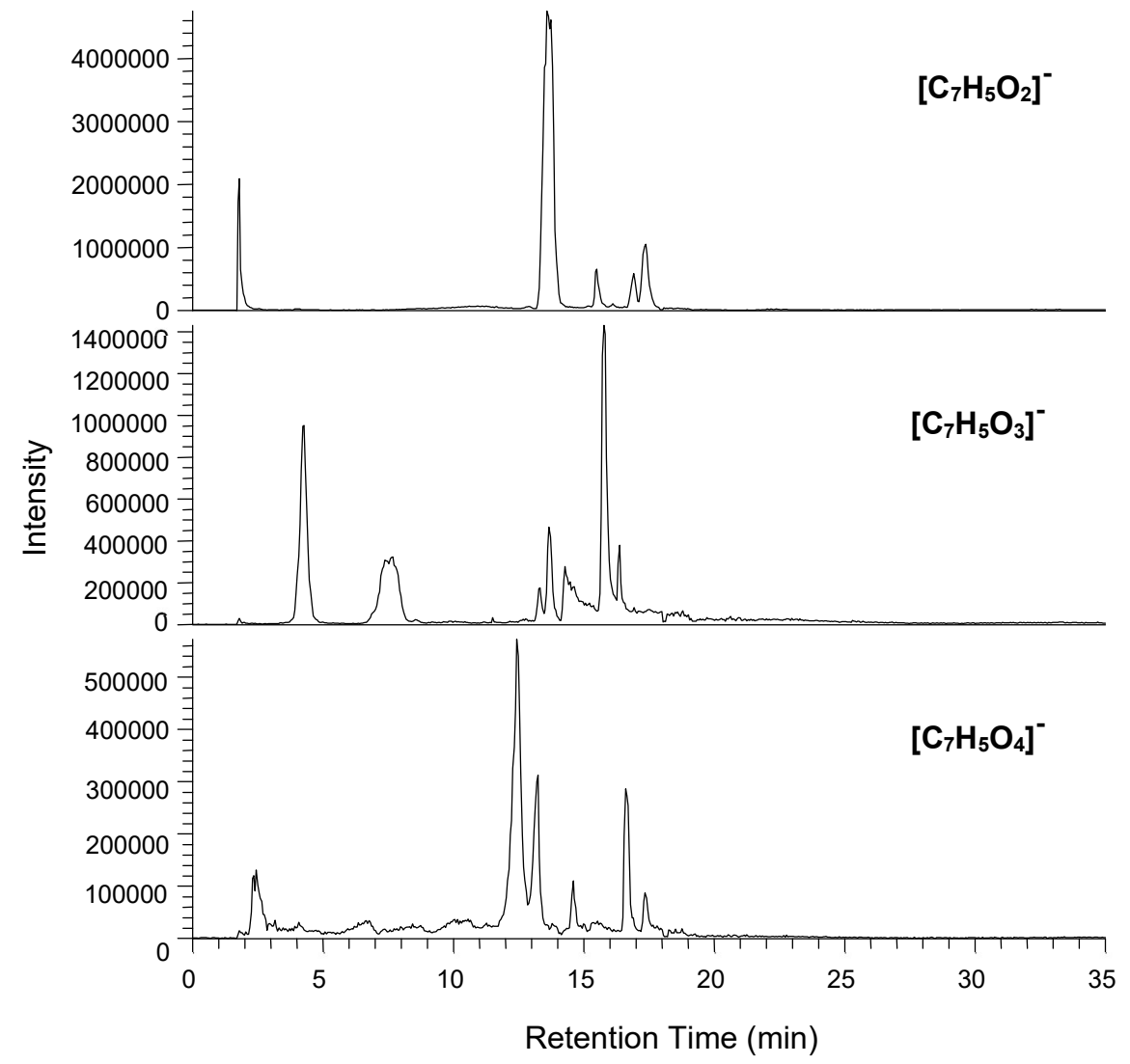

Figure S3: Extracted LC chromatograms of several CHO ions including m/z 121.0293 $\left(\left[\mathrm{C}_{7} \mathrm{H}_{5} \mathrm{O}_{2}\right]^{-}\right), 137.0243\left(\left[\mathrm{C}_{7} \mathrm{H}_{5} \mathrm{O}_{3}\right]^{-}\right)$and $153.0192\left(\left[\mathrm{C}_{7} \mathrm{H}_{5} \mathrm{O}_{4}\right]^{-}\right)$from one representative sample (HBDM-1). 


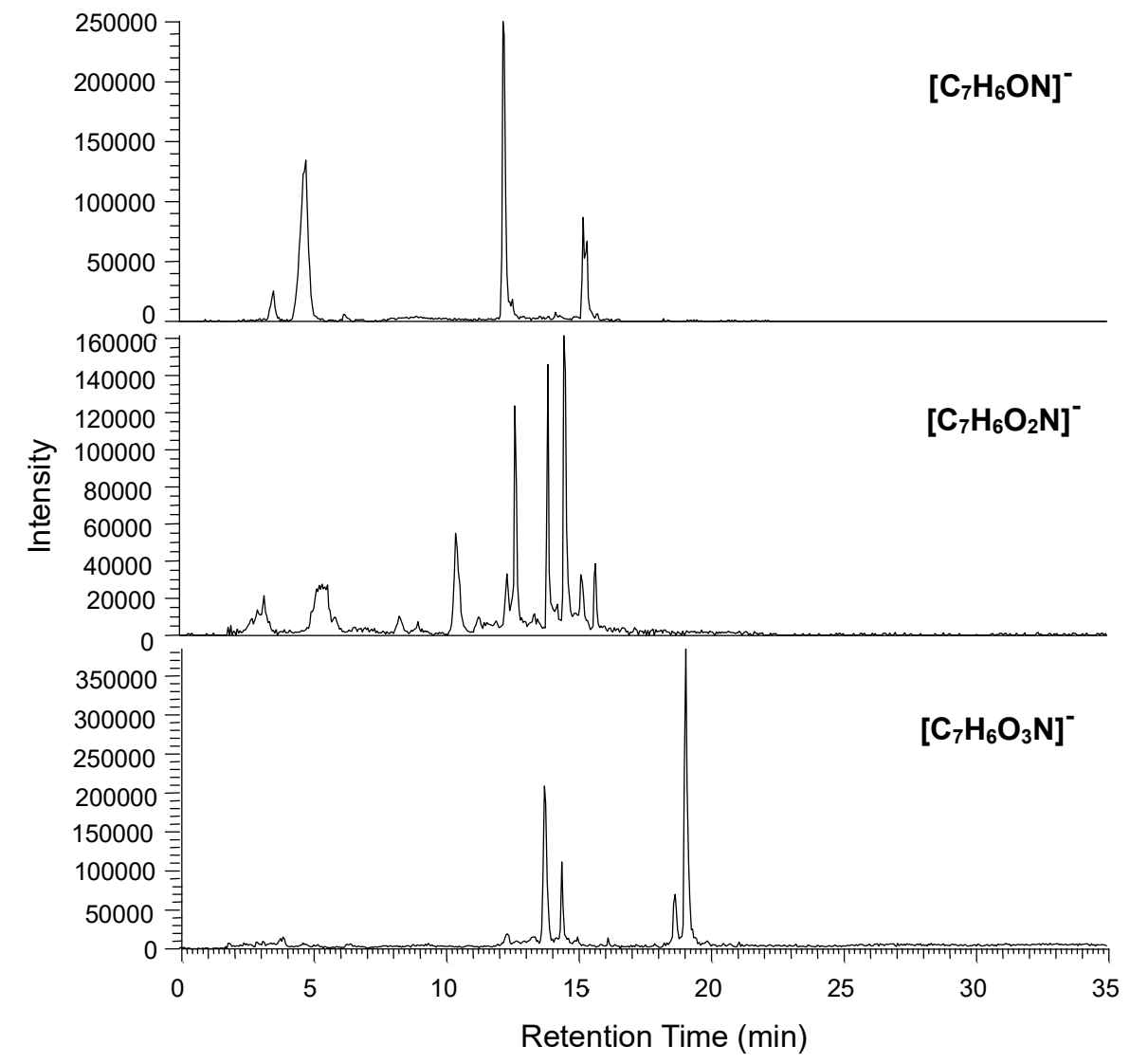

Figure S4: Extracted LC chromatograms of several CHON ions including $\mathrm{m} / \mathrm{z}$ $120.0453\left(\left[\mathrm{C}_{7} \mathrm{H}_{6} \mathrm{ON}\right]^{-}\right), 136.0403\left(\left[\mathrm{C}_{7} \mathrm{H}_{6} \mathrm{O}_{2} \mathrm{~N}\right]^{-}\right)$and $152.0352\left(\left[\mathrm{C}_{7} \mathrm{H}_{6} \mathrm{O}_{3} \mathrm{~N}\right]^{-}\right)$from one representative sample (HBDM-1). 


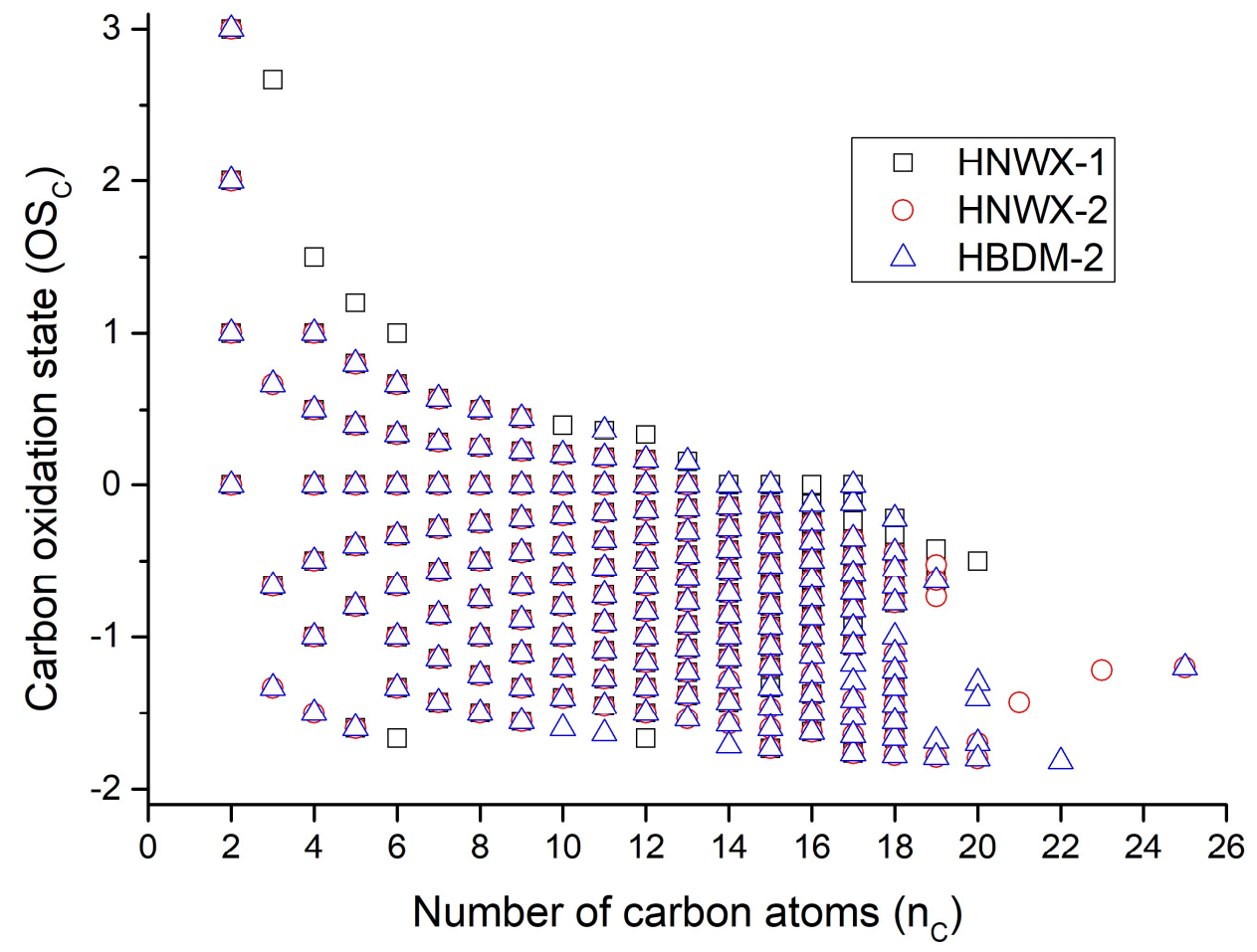

Figure S5: The distribution of carbon oxidation state $\left(\mathrm{OS}_{\mathrm{C}}\right)$ for $\mathrm{CHO}$ molecules in HNWX-1, HNWX -2 and HBDM-2 samples. 

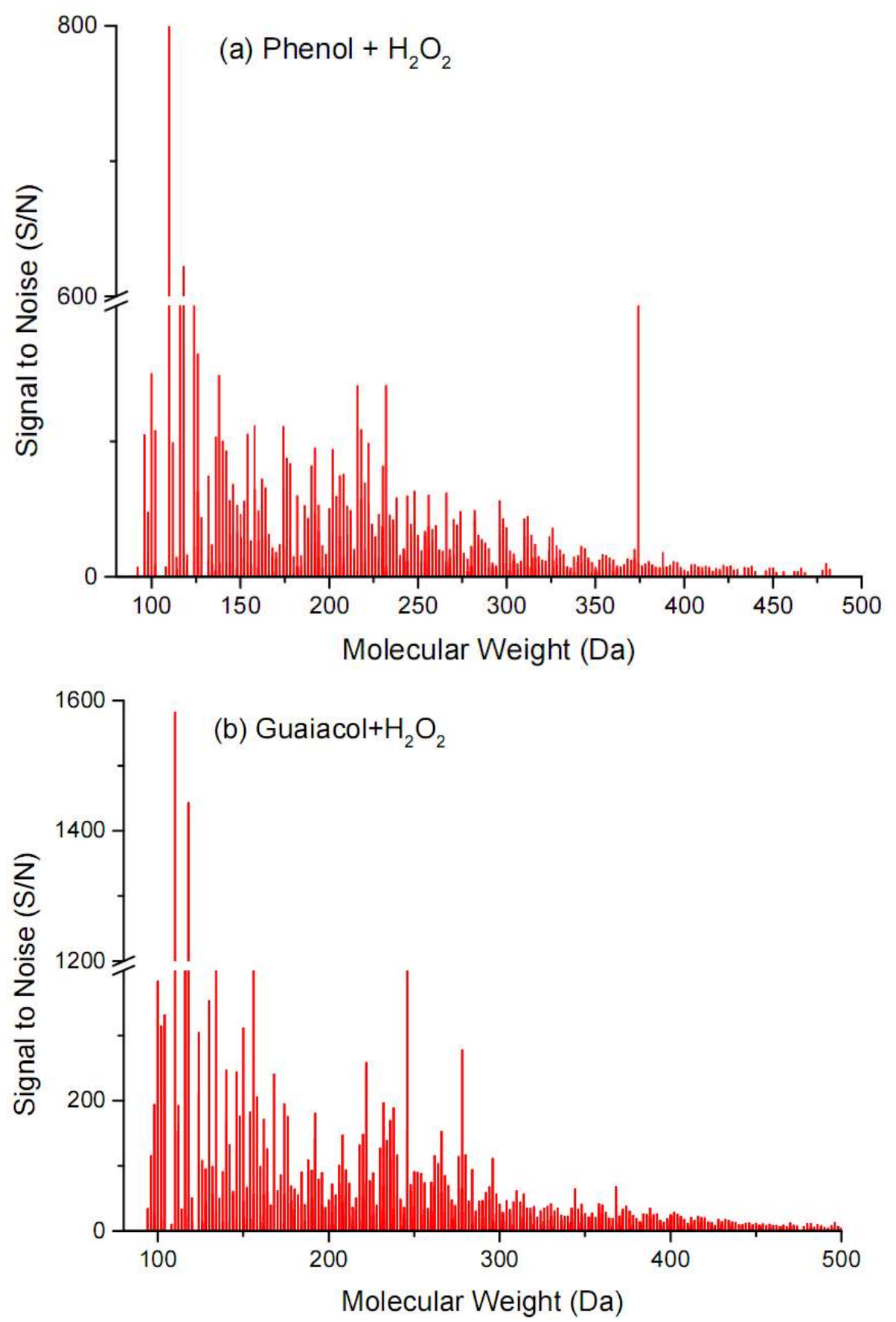

Figure S6: Reconstructed mass spectra of photochemical products of (a) phenol and (b) guaiacol by $\mathrm{OH}$ reactions in the aqueous phase, respectively. 


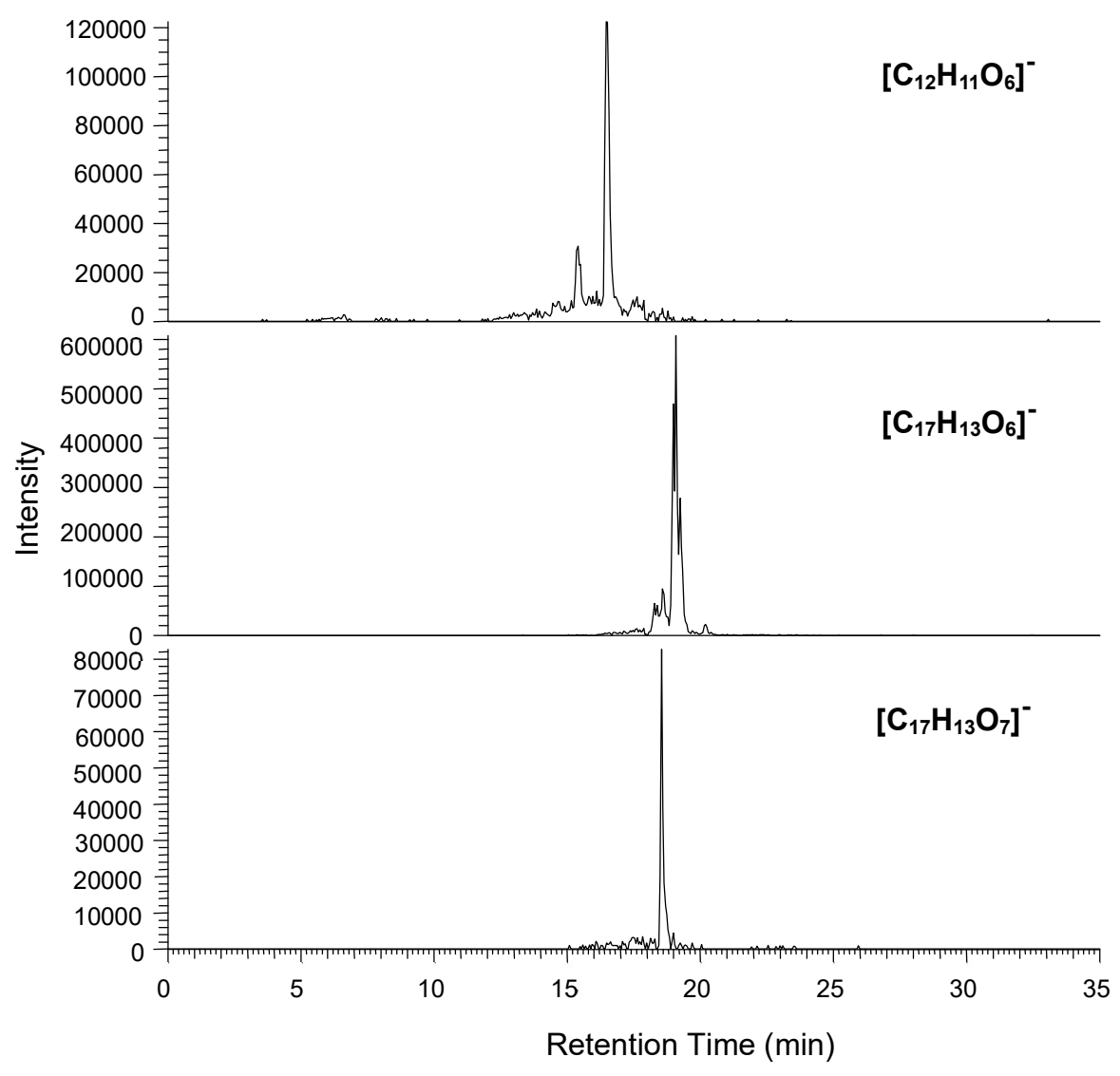

Figure S7: Extracted LC chromatograms of m/z $251.0564\left(\left[\mathrm{C}_{12} \mathrm{H}_{11} \mathrm{O}_{6}\right]^{-}\right), 313.0724$ $\left(\left[\mathrm{C}_{17} \mathrm{H}_{13} \mathrm{O}_{6}\right]^{-}\right)$and $329.0674\left(\left[\mathrm{C}_{17} \mathrm{H}_{13} \mathrm{O}_{7}\right]^{-}\right)$from one representative sample (HBDM-1). 

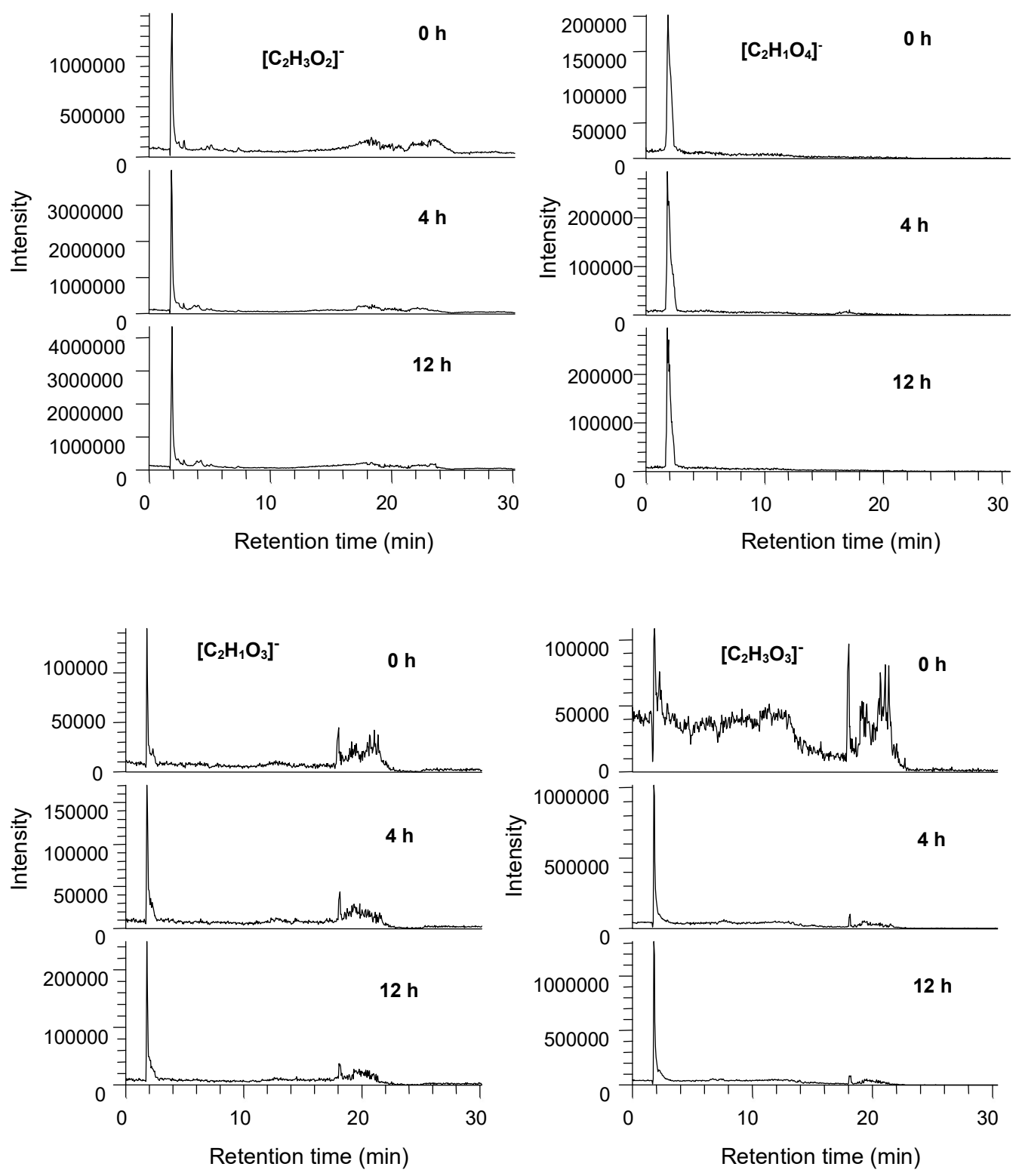

Figure S8: Extracted LC chromatograms of $\left[\mathrm{C}_{2} \mathrm{H}_{3} \mathrm{O}_{2}\right]^{-},\left[\mathrm{C}_{2} \mathrm{H}_{1} \mathrm{O}_{4}\right]^{-},\left[\mathrm{C}_{2} \mathrm{H}_{1} \mathrm{O}_{3}\right]^{-}$and $\left[\mathrm{C}_{2} \mathrm{H}_{3} \mathrm{O}_{3}\right]^{-}$from one representative sample (HBDM-1) taken in the dark (0h) and after $4 \mathrm{~h}$ and $12 \mathrm{~h}$ of irradiation. 

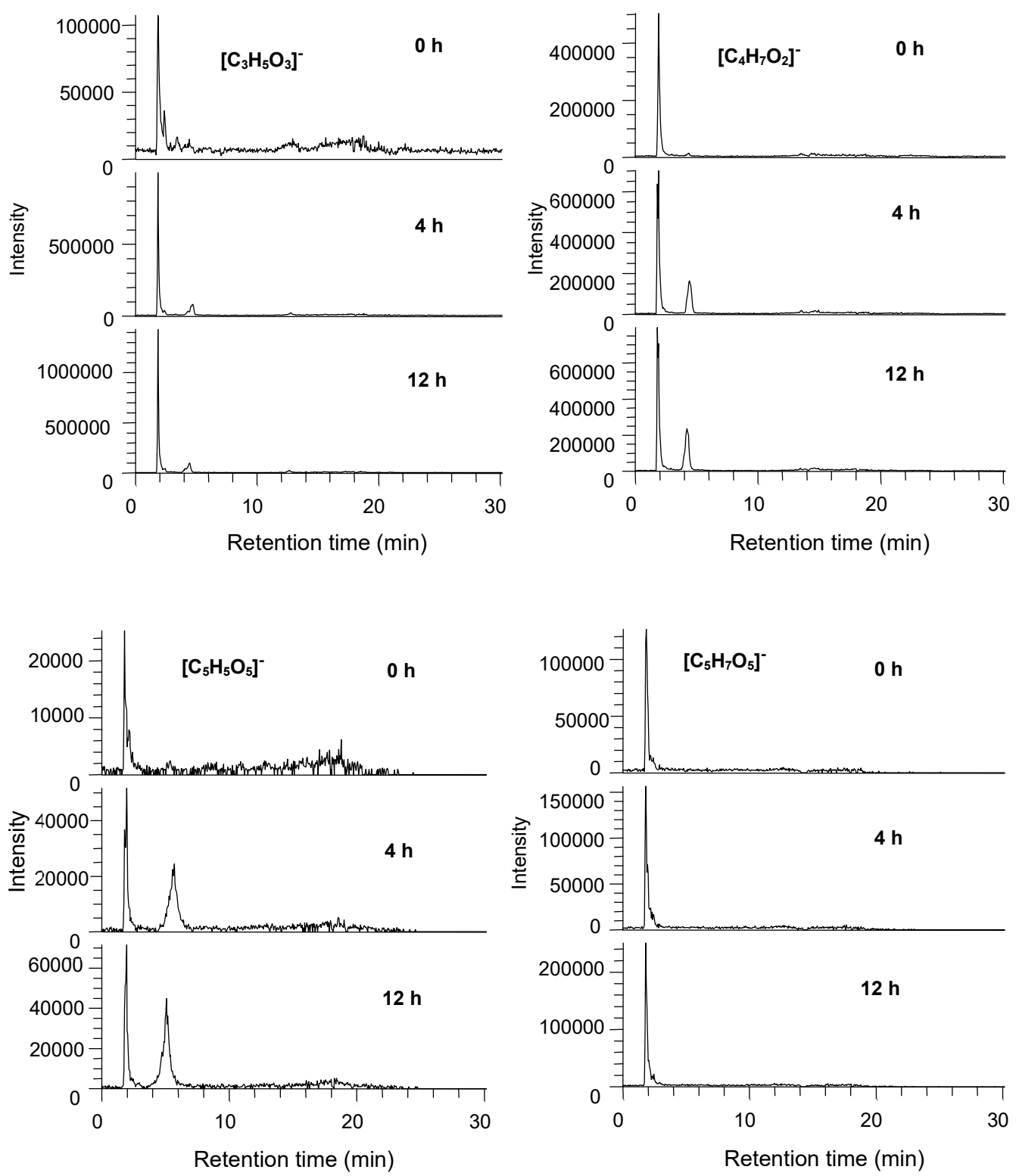

Figure S9: Extracted LC chromatograms of $\left[\mathrm{C}_{3} \mathrm{H}_{5} \mathrm{O}_{3}\right]^{-}$, $\left[\mathrm{C}_{4} \mathrm{H}_{7} \mathrm{O}_{2}\right]^{-},\left[\mathrm{C}_{5} \mathrm{H}_{5} \mathrm{O}_{5}\right]^{-}$and $\left[\mathrm{C}_{5} \mathrm{H}_{7} \mathrm{O}_{5}\right]^{-}$from one representative sample (HBDM-1) taken in the dark (0h) and after $4 \mathrm{~h}$ and $12 \mathrm{~h}$ of irradiation. 


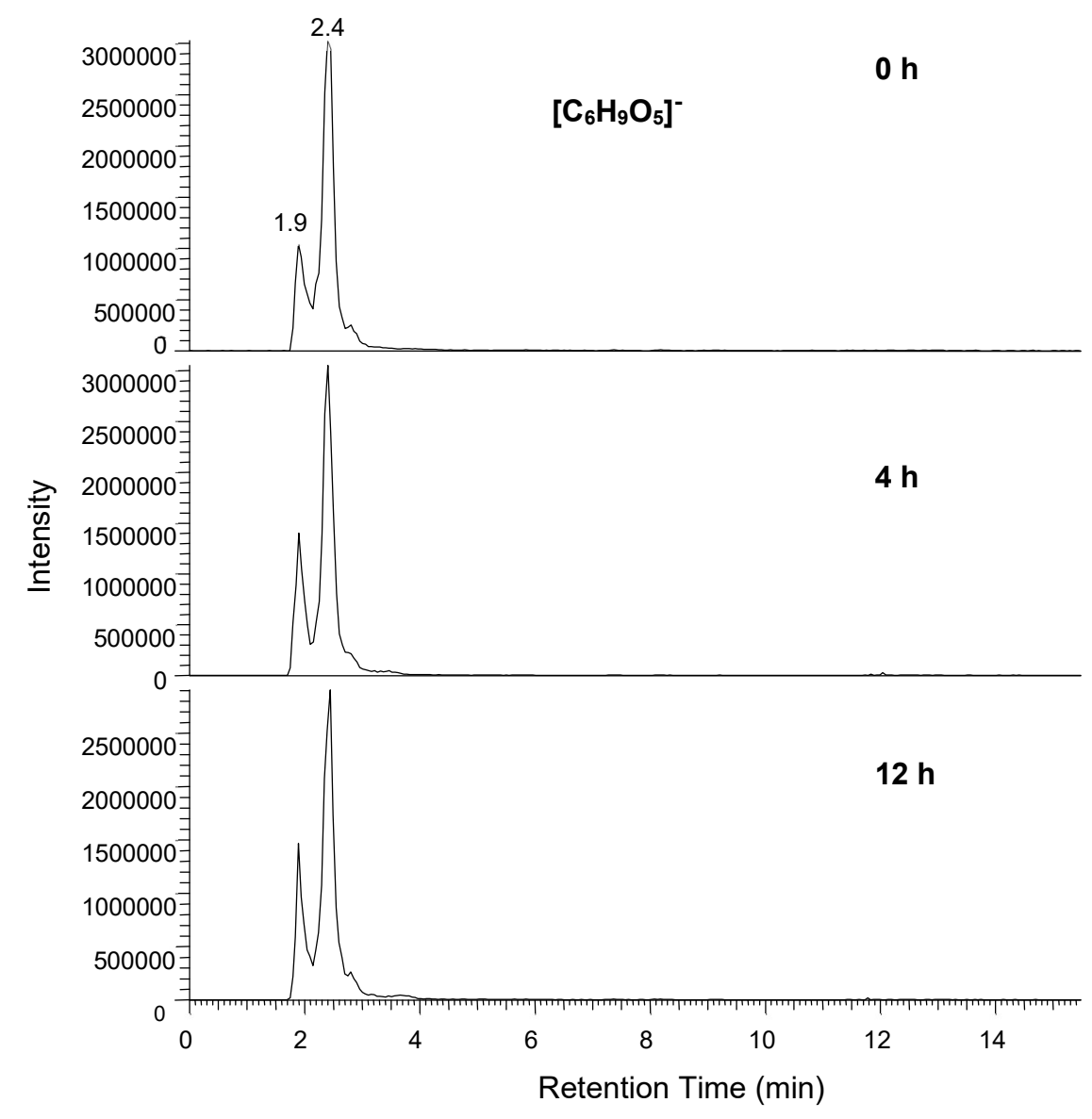

Figure S10: Extracted LC chromatograms of $\mathrm{m} / \mathrm{z}=161.0454\left(\left[\mathrm{C}_{6} \mathrm{H}_{9} \mathrm{O}_{5}\right]^{-}\right)$from one representative sample (HBDM-1) taken in the dark (0h) and after $4 \mathrm{~h}$ and $12 \mathrm{~h}$ of irradiation. 


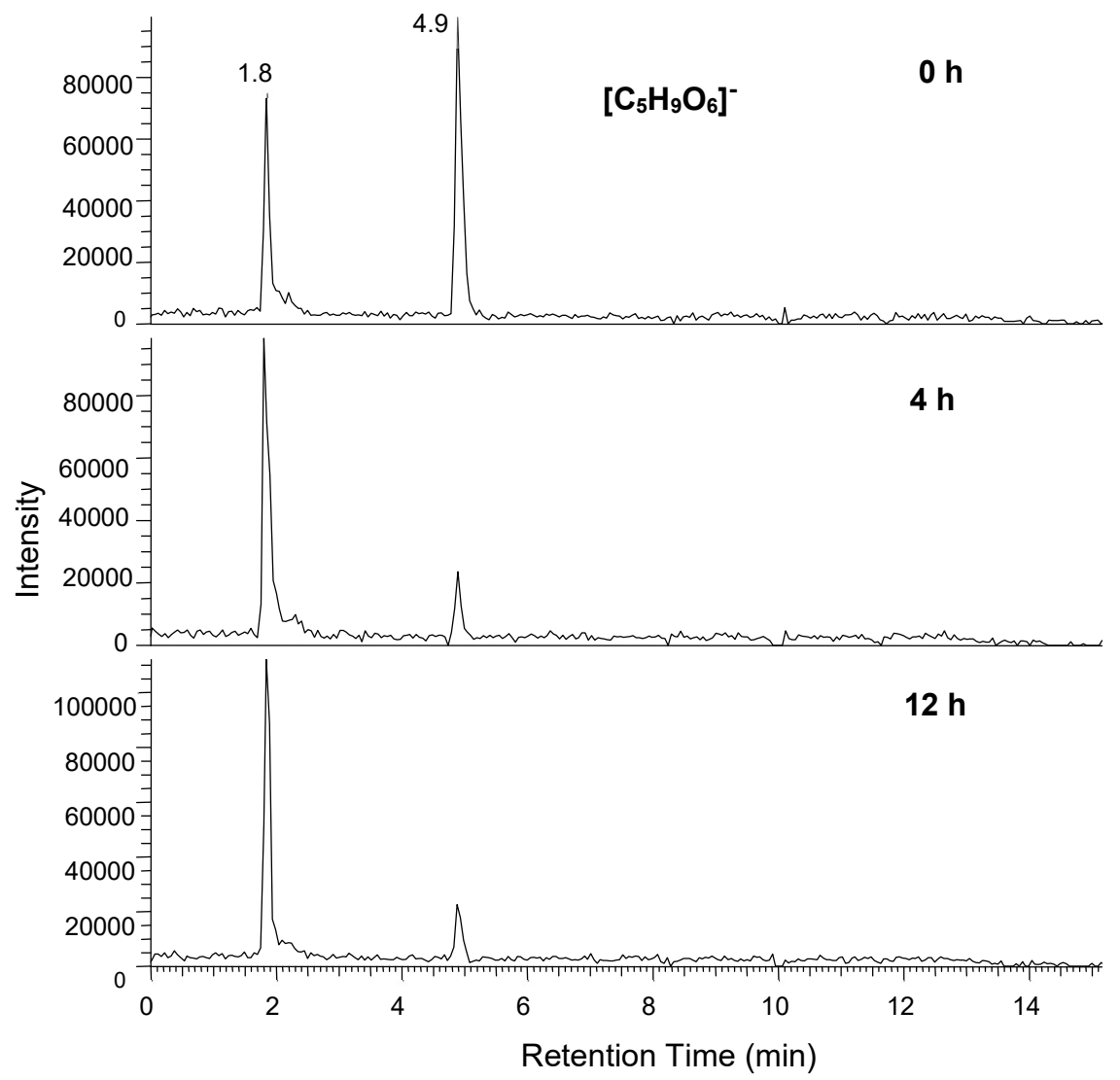

Figure S11: Extracted LC chromatograms of m/z $165.0405\left(\left[\mathrm{C}_{5} \mathrm{H}_{9} \mathrm{O}_{6}\right]^{-}\right)$from one representative sample (HBDM-1) taken in the dark (0h) and after $4 \mathrm{~h}$ and $12 \mathrm{~h}$ of irradiation. 


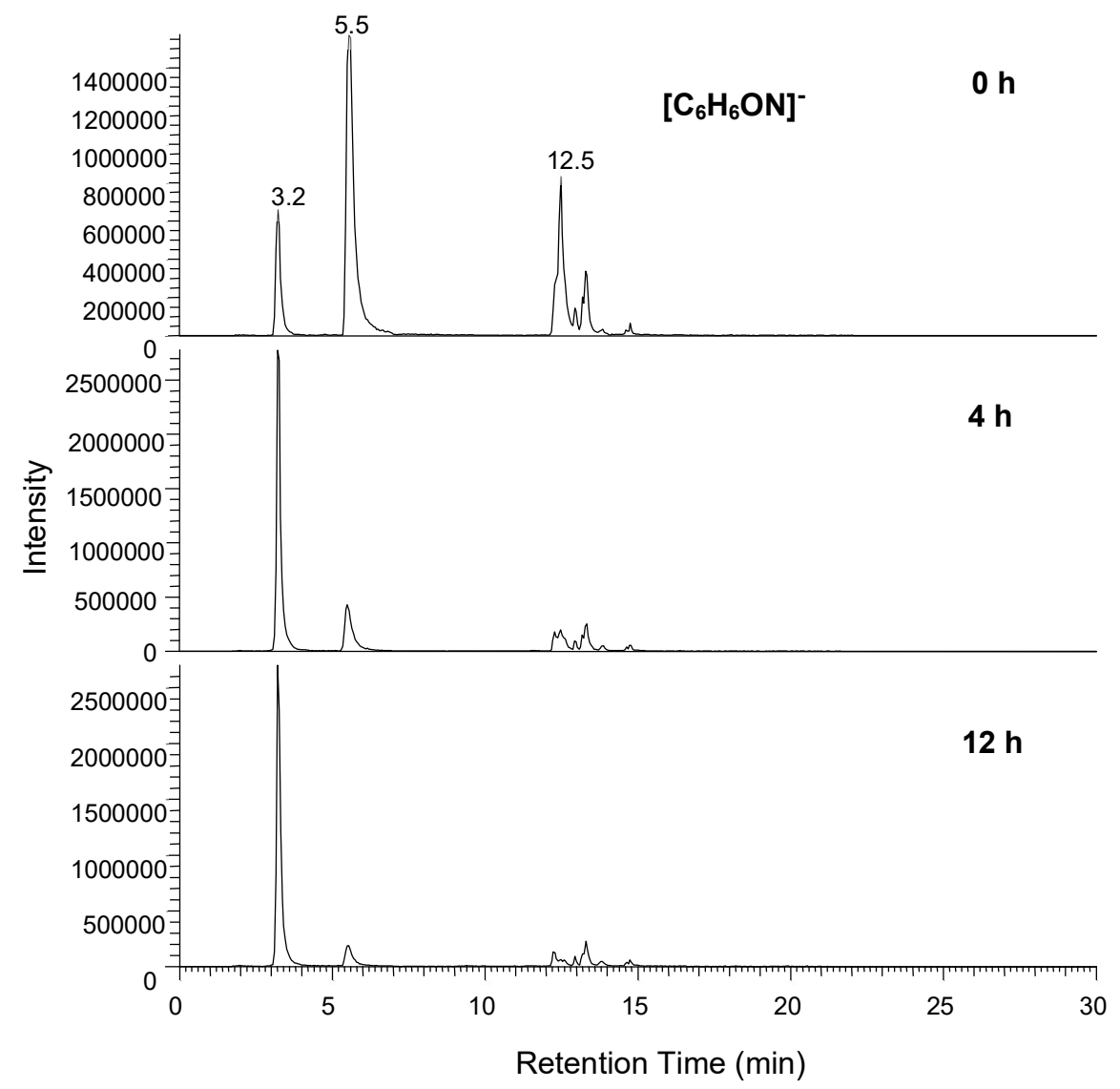

Figure S12: Extracted LC chromatograms of m/z $108.0453\left(\left[\mathrm{C}_{6} \mathrm{H}_{6} \mathrm{ON}\right]^{-}\right)$from one representative sample (HBDM-1) taken in the dark (0h) and after $4 \mathrm{~h}$ and $12 \mathrm{~h}$ of irradiation. 